\title{
A dual characterization of incentive efficiency
}

\author{
Belén Jerez* \\ Departamento de Economía, Universidad Carlos III de Madrid, Getafe, Madrid 28903, Spain
}

\begin{abstract}
We show that incentive efficient allocations in economies with adverse selection and moral hazard problems can be determined as optimal solutions to a linear programming problem and we use duality theory to obtain a complete characterization of the optima. Our dual analysis identifies welfare effects associated with the incentives of the agents to truthfully reveal their private information. Because these welfare effects may generate non-convexities, incentive efficient allocations may involve randomization. Other properties of incentive efficient allocations are also derived.
\end{abstract}

JEL classification: D50; C61; D82

Keywords: Asymmetric information; Incentive efficiency; Linear programming; Duality

\section{Introduction}

It is well known that informational asymmetries generate adverse selection and moral hazard problems. To take these problems into account, in addition to the standard resource constraints, an allocation must satisfy incentive compatibility constraints: agents must be given incentives to truthfully reveal their private characteristics and actions. Adapting the well-established techniques of linear programming and duality theory to characterize efficient allocations under asymmetric information, we study the role of incentive compatibility in determining allocations.

We introduce the linear programming methodology using two simple economies. The first is an adverse selection insurance economy similar to the one used by

\footnotetext{
*Corresponding author. Fax: + 34-91-624-9875

E-mail address: mjerez@eco.uc3m.es.
} 
Rothschild and Stiglitz [28] and Wilson [30]. The second is a moral hazard version of the first. In both cases, we show that incentive efficient allocations (i.e. allocations which are Pareto optimal in the set of resource feasible and incentive compatible allocations) can be determined as solutions to a linear programming problem. Then we use the primal problem, its dual, and their corresponding complementary slackness conditions to obtain a precise and simple characterization of these allocations.

The adverse selection model is a standard insurance economy with a continuum of agents of two types, high-and-low risk, and two possible idiosyncratic endowment states. Following Prescott and Townsend [26], we define allocations in the space of lotteries over bundles of contingent commodities. A lottery is just a random insurance plan. We assume that contingent claims are perfectly verifiable and fully enforceable. ${ }^{1}$ Agents have von Neumann-Morgenstern preferences. Therefore, their objective function as well as their incentive constraints are linear in the lotteries. Incentive efficient allocations can then be determined as optimal solutions to a linear programming problem; more precisely, a Linear Semi-Infinite Programming problem. We derive the "dual problem" and use the complementary slackness theorem to characterize the set of incentive efficient allocations. We also show that there is no loss of generality in restricting attention to lotteries with finite support. Then we derive properties of incentive efficient allocations as well as conditions under which lotteries can be dispensed with.

The main economic insights of the analysis arise from the use of duality theory. In the "dual problem" we identify the welfare effects arising from constraints on the allocation. Apart from the standard welfare effects (i.e., utilities and economic costs) we find others associated with the incentives of the agents to reveal their risk types. Intuitively, a given allocation may be relatively costly because it gives a greater incentive to one type of agent to misrepresent their type. For instance, all actuarially fair insurance plans for the low-risk agents generate identical economic costs. However, those plans that are more attractive to the high-risk agents imply higher total welfare costs. The reason is that under such plans it becomes more costly to prevent the high-risk agents from lying about their type (i.e., more resources are needed to induce truthful revelation).

Incentive efficient allocations must internalize the welfare costs of incentives. Our analysis shows that these welfare costs are not convex. Hence, some of the incentive efficient allocations may be random. Randomization may be used to optimally separate types with different degrees of risk aversion. For instance, randomization is beneficial when low-risk agents are risk neutral and high-risk agents are risk averse. In this case, any fair insurance plan is equally good for a low-risk agent and equally costly in terms of resources. Yet a random insurance plan implies lower total welfare costs because it involves greater risk and is thus less attractive to the high-risk agents. In general, whenever the incentives of the high-risk agents are at issue, and as long as these agents are sufficiently more risk averse than low-risk agents, assigning a lottery

\footnotetext{
${ }^{1}$ Bisin and Gottardi [4] and Bisin and Guatoli [6] depart from this "exclusive" benchmark and study economies with non-verifiable trades, while Dubey et al. [10] study environments where asymmetric information arises from the possibility of default.
} 
to the latter will reduce the welfare cost of incentives and may allow a welfare improvement. By contrast, if low-risk agents are at least as risk averse as high-risk agents then the optimal insurance plan of the low-risk agents is deterministic.

The analysis of the moral hazard economy is very similar. In this economy, there is a continuum of ex ante identical agents and two possible idiosyncratic endowment states. Each agent can exert either high or low effort at a direct utility cost. Higher effort reduces the probability of ending up in the poor state. The main difference compared with the adverse selection model is that allocations may now involve two kinds of randomization: not only a random insurance plan, but also a random effort level. Our dual analysis identifies welfare effects arising from the incentive constraints on the allocation. Suppose an allocation assigns an insurance plan to the agents and specifies high effort. The total welfare cost of the assignment depends on how willing the agents are to conform to the high-effort specification. We show that insurance plans that give the agents a greater incentive to shirk imply higher total welfare costs. We also show that if risk aversion decreases fast enough with effort then optimal insurance plans are random. On the other hand, effort may also be random. When their effort is high, agents have higher expected wealth, but this comes at a direct utility cost. In some instances, agents may be willing to give up some consumption to reduce their effort. The tradeoff between consumption and effort is resolved by allowing the agents to provide low effort with some positive probability at the cost of reducing their expected consumption. We find that if the agents' expected wealth is large enough, or if the cost of effort increases fast enough with consumption, incentive efficient allocations involve random effort.

\subsection{Related literature}

Harris and Townsend [16] study resource allocation problems in a large class of environments with private information concerning the agents' preferences. They show that the equilibrium outcome of any general allocation mechanism can be replicated using a direct revelation mechanism that induces truth telling (see also Myerson [23]). Moreover, an allocation is optimal if and only if it is associated with an incentive efficient direct revelation mechanism. Myerson [24] extends these results to general Bayesian games with incomplete information. Our contribution is to exploit the linear structure which, as Myerson [24] shows, is inherent to these environments. Adapting the techniques of linear programming and duality theory, we obtain a complete characterization of the set of incentive efficient allocations for two standard economies with adverse selection and moral hazard. In addition, duality emerges as a powerful tool which clarifies the role of incentive compatibility in determining allocations. Our analysis is related to the work of Myerson [25] on bargaining under incomplete information. The bargaining solution in Myerson [25] is a direct mechanism that is incentive efficient and equitable when interpersonal comparisons are made using some "virtual utility" scales for the players. A player's virtual utility differs from her real utility. In particular, the player's virtual utility exaggerates the differences between her preferences and the preferences of other players who may try to report type of the player instead of truthfully revealing their 
own types. Myerson shows that incentive efficient mechanisms satisfy a linear programming problem. Virtual utility is defined so that the Lagrangian function of this problem can be written as the expected sum of the players' virtual utilities. Then in an efficient agreement players behave as if their objective was to maximize their virtual, instead of their real utilities.

The idea that lotteries may enhance welfare by separating types on the basis of their attitude towards risk is discussed by Prescott and Townsend [26,27], and further investigated by Cole [9] and Arnott and Stiglitz [2]. We show that, when types have different preferences for risk, lotteries enhance efficiency by reducing the welfare costs of incentives. In recent work, Kehoe et al. [20] study an exchange economy with private information about endowments where agents purchase insurance before their endowments are realized. They show that if the agents' preferences display decreasing absolute risk aversion then lotteries are suboptimal. In their set-up, agents with high-endowment realizations may have incentives to report a low endowment in order to receive an insurance compensation. Assigning a random insurance plan to agents who report a low endowment helps reduce this incentive only if high-endowment agents are more risk averse than low-endowment agents, that is, if utility exhibits increasing absolute risk aversion. We obtain analogous results for the adverse selection and moral hazard economies: if the type with the highest average consumption has higher absolute risk aversion (or if absolute risk aversion does not decrease with effort) then lotteries are suboptimal. With adverse selection, assigning a random insurance plan to the type with the highest expected consumption helps prevent misrepresentation by the other type only if the latter is more risk averse. Similarly, with moral hazard, assigning a random insurance plan when high effort is specified helps prevent a deviation to low effort only if risk aversion decreases with effort.

Our approach is inspired by the work of Makowski and Ostroy [22]. These authors use a linear programming model to study large economies with full information. Gretsky et al. [14] present a similar linear programming treatment of large assignment economies. This work is a first step in trying to introduce incentive constraints in those models.

The structure of the paper is as follows. In Section 2, we present the adverse selection model. We set up the linear programming problem and its dual. Then we use the complementary slackness theorem to characterize incentive efficient allocations and study their properties. Section 3 presents a similar analysis for the case of moral hazard. The proofs are deferred to the Appendix which involves an application of Linear Semi-Infinite Programming.

\section{Adverse selection}

\subsection{The economy}

Consider an exchange economy with a single consumption good and a continuum of agents of two types $i=L, H$. The fraction of agents of type $i$ is denoted by $\xi_{i}$. 
The agents in the economy are subject to idiosyncratic endowment shocks. Specifically, each agent can be in one of two states $s=1,2$. At each state, the agent is endowed with a different amount $\omega_{s}$ of the good, where $0<\omega_{1}<\omega_{2}$. The probability that state 1 (the low-endowment state) is realized is higher for an agent of type $H$ ("high risk") than for an agent of type $L$ ("low risk"). These probabilities will be denoted by $\theta_{H}$ and $\theta_{L}$, respectively, so that $0<\theta_{L}<\theta_{H}<1$. Agents of type $i$ have von Neumann-Morgenstern preferences over contingent consumption plans as defined by the Bernoulli utility function $U_{i}: \mathbb{R}_{+} \rightarrow \mathbb{R}$, where $U_{i}$ is twice continuously differentiable, strictly increasing, and strictly concave with $\lim _{c \rightarrow 0} U_{i}^{\prime}(c)=\infty$ and $\lim _{c \rightarrow \infty} U_{i}^{\prime}(c)=0 .^{2}$

Idiosyncratic shocks are independent across agents and render no uncertainty at the aggregate level. ${ }^{3}$ Ex post, the fraction of type- $i$ agents with a low endowment is $\theta_{i}$, and the average endowment of the type- $i$ group is $\bar{\omega}_{i}=\theta_{i} \omega_{1}+\left(1-\theta_{i}\right) \omega_{2}$. The expost aggregate endowment is given by $\bar{\omega}=\xi_{L} \bar{\omega}_{L}+\left(1-\xi_{L}\right) \bar{\omega}_{H}$.

Agents choose their contingent consumption plans before the realization of the endowment shock. The structure of individual uncertainty is common knowledge and the realization of the endowment shocks is observable. State-contingent net trades are perfectly verifiable and fully enforceable ex post. However, an individual agent's type is known only to herself.

\subsection{Allocations}

In this section, we define the space of allocations and describe allocations which are physically feasible and incentive compatible. Then we define incentive efficient allocations.

Let $Z$ denote the net trade set of an agent; that is, the set of all pairs $z=$ $\left(z_{1}, z_{2}\right) \in \mathbb{R}^{2}$ such that $z_{s} \geqslant-\omega_{s}$ for $s=1,2$. For any $z \in Z$, the expected net trade of

\footnotetext{
${ }^{2}$ The model is slightly more general than the Rothschild-Stiglitz [28] economy, where utilities are typeinvariant. As we will see, differences in tastes across types may have important consequences for the nature of the incentive efficient allocations. All results can be extended to state-dependent utilities and to any finite number of idiosyncratic states.

${ }^{3} \mathrm{We}$ assume that the law of large numbers holds. It is well known that in the standard continuum model, where the set of agents is modeled as the unit interval with Lebesgue measure, the law of large numbers sometimes cannot be stated due to a measurability problem and may not be true even when there is no measurability problem (see Judge [19]). In fact, in the standard measure theoretic framework it not possible to obtain non-trivial processes of i.i.d. random variables that yield the "exact" law of large numbers (see Sun [29]). For this reason, the usual continuum model is not a good approximation of the limit of a sequence of large finite economies with independent shocks across households. An ideal limit model can be constructed, however, using hyperfinite models from non-standard analysis. Sun [29] constructs a non-standard representation with a continuum of agents indexed by a hyperfinite Loeb measure space where any measurable process for the endowments that preserves independence for almost all pairs yields the exact law of large numbers (see Sun [29, Theorem 3.10, p. 436]). The non-standard model is asymptotically implementable in a setting with a large but finite number of agents so, in Sun's words, it is "elementarily equivalent" to the standard model. For alternative approaches to this problem see Feldman and Gilles [11], and Hammond and Lisboa [15].
} 
an agent of type $i$ is given by

$$
r_{i}\left(z_{1}, z_{2}\right)=\theta_{i} z_{1}+\left(1-\theta_{i}\right) z_{2}
$$

and her expected utility is defined as

$$
E U_{i}\left(z_{1}, z_{2}\right)=\theta_{i} U_{i}\left(\omega_{1}+z_{1}\right)+\left(1-\theta_{i}\right) U_{i}\left(\omega_{2}+z_{2}\right)
$$

An allocation in this economy is a random net trade assignment for each type. That is, before the realization of individual uncertainty, each agent receives a lottery depending on her type. The realization of this lottery determines a net trade, and thus, a contingent consumption plan for the agent. Formally, an allocation is a pair of probability measures on $Z$. Denote the space of Borel measures on $Z$ which have compact support by $M_{\mathrm{c}}(Z)$. The space of allocations is the set of pairs $\left(x_{L}, x_{H}\right) \in M_{\mathrm{c}}(Z) \times M_{\mathrm{c}}(Z)$ such that

$$
\int_{Z} d x_{i}=1, \quad x_{i} \geqslant 0, \quad i=L, H^{4}
$$

Here, $x_{i}$ is a probability measure describing the lottery assigned to each agent of type $i$. That is, for any Borel set $B \subset Z, x_{i}(B)$ is the probability that the agent is assigned a net trade $z \in B$. Under this formulation, deterministic assignments are given by degenerate measures. We assume that lotteries are assigned across agents so as to preserve aggregate certainty. ${ }^{5}$ Then $x_{i}$ is also the distribution of net trades of type- $i$ agents once the outcomes of all individual lotteries are realized (e.g. $x_{i}(B)$ is the fraction of agents of type $i$ assigned to a net trade $z \in B$ ).

An allocation is feasible if the aggregate net trade is non-positive. The average net trade of the agents of type $i$ is given by $\left\langle r_{i}, x_{i}\right\rangle=\int_{Z} r_{i} d x_{i}$. Hence, the aggregate resource constraint is

$$
\xi_{L}\left\langle r_{L}, x_{L}\right\rangle+\left(1-\xi_{L}\right)\left\langle r_{H}, x_{H}\right\rangle \leqslant 0 .
$$

Since types are private information, an agent of type $i$ may claim to be any of the two types $j=1,2$, and receive expected utility $\left\langle E U_{i}, x_{j}\right\rangle=\int_{Z} E U_{i} d x_{j}$. An allocation is incentive compatible if it is not in the interest of agents to misrepresent their type:

$$
\left\langle E U_{i}, x_{i}\right\rangle \geqslant\left\langle E U_{i}, x_{j}\right\rangle, \quad j \neq i, \quad i=L, H .
$$

An allocation is incentive efficient if it is feasible, incentive compatible, and there exists no other feasible and incentive compatible allocation that is weakly preferred by both types and strictly preferred by at least one type.

\footnotetext{
${ }^{4}$ This description of the space of allocations is equivalent to the one used by Prescott and Townsend [26]. A formal difference is that in their model consumption sets are finite, so lotteries are essentially elements in the Euclidean space.

${ }^{5}$ Since lotteries need not be independent across agents, it is easy to construct a standard process of random net trade assignments for the agents that yields no aggregate uncertainty (see Feldman and Gilles [11]). Therefore, the definition of the space of allocations is consistent with the assumption of aggregate certainty. I would like to thank one of the referees for pointing this out to me.
} 


\subsection{The primal and dual problems}

In this section, we show that every incentive efficient allocation is an optimal solution to a linear programming problem.

The problem of the planner is to find an allocation so as to maximize a weighted average of the utilities of the two types subject to the feasibility and the incentive constraints. Note that utilities are linear in the lotteries. Constraints (2.1)-(2.3) are also linear. In order to extend the inner product notation $\langle\cdot, \cdot\rangle$ to the adding-up constraint (2.1), we define $\mathscr{I}: Z \rightarrow\{0,1\}$ to be the characteristic function on $Z$ and write $\left\langle\mathscr{I}, x_{i}\right\rangle=\int_{Z} d x_{i}$ for $i=L, H$. For given positive welfare weights $\left(\gamma_{L}, \gamma_{H}\right)$, with $\gamma_{H}=1-\gamma_{L}$, the problem of the planner is to find an allocation $\left(x_{L}, x_{H}\right) \in M_{\mathrm{c}}(Z) \times M_{\mathrm{c}}(Z)$ to solve

(D) $\sup \gamma_{L}\left\langle E U_{L}, x_{L}\right\rangle+\left(1-\gamma_{L}\right)\left\langle E U_{H}, x_{H}\right\rangle$

s.t.

$$
\begin{aligned}
& \left\langle\mathscr{I}, x_{L}\right\rangle=1, \\
& \left\langle\mathscr{I}, x_{H}\right\rangle=1, \\
& -\left\langle E U_{L}, x_{L}\right\rangle+\left\langle E U_{L}, x_{H}\right\rangle \leqslant 0, \\
& \left\langle E U_{H}, x_{L}\right\rangle-\left\langle E U_{H}, x_{H}\right\rangle \leqslant 0, \\
& \xi_{L}\left\langle r_{L}, x_{L}\right\rangle+\left(1-\xi_{L}\right)\left\langle r_{H}, x_{H}\right\rangle \leqslant 0, \\
& x_{L}, x_{H} \geqslant 0 .
\end{aligned}
$$

Problem $(D)$ is a linear programming problem. Standard results in linear programming theory show that problem $(D)$ is dual to another linear programming problem, known as the primal problem or problem $(P)$. Whereas problem $(D)$ is a maximization problem with an infinite number of variables and a finite number of constraints, problem $(P)$ is a minimization problem with a finite number of variables and an infinite number of constraints. In optimization theory, these kind of problems are known as Linear Semi-Infinite Programming (LSIP) problems. ${ }^{6}$ As we shall see, the primal and dual problems are related because the primal variables are also the shadow prices of the dual constraints, and vice versa.

Problem $(P)$, which is derived in detail in Appendix A, consists of finding a quintuple $\left(\alpha_{L}, \alpha_{H}, \beta_{L}, \beta_{H}, q\right) \in \mathbb{R}^{5}$ to solve

\footnotetext{
${ }^{6}$ An LSIP problem is an optimization problem with linear objective and linear constraints in which either number of variables or the number of constraints is finite. For an excellent survey on LSIP theory, see Goberna and López [12].
} 
(P) inf $\alpha_{L}+\alpha_{H}$

s.t.

$$
\begin{aligned}
& \alpha_{L} \geqslant \gamma_{L} E U_{L}(z)+\beta_{L} E U_{L}(z)-\beta_{H} E U_{H}(z)-q \xi_{L} r_{L}(z) \\
& \forall z \in Z \\
& \alpha_{H} \geqslant\left(1-\gamma_{L}\right) E U_{H}(z)-\beta_{L} E U_{L}(z)+\beta_{H} E U_{H}(z)-q\left(1-\xi_{L}\right) r_{H}(z) \\
& \forall z \in Z \\
& \\
& \beta_{L}, \beta_{H}, q \geqslant 0
\end{aligned}
$$

where $\left(\alpha_{L}, \alpha_{H}\right)$ are the shadow prices of the adding-up constraints (2.4) and (2.5), $\left(\beta_{L}, \beta_{H}\right)$ are the shadow prices of the incentive constraints (2.6) and (2.7), and $q$ is the shadow price of the resource constraint (2.8).

Denote the optimal values for problems $(P)$ and $(D)$ by $v(P)$ and $v(D)$, respectively. It is easy to see that both problems are consistent (i.e. their feasible sets are not empty) as well as bounded (i.e. $v(P)$ and $v(D)$ are finite). ${ }^{7}$ However, unlike an ordinary linear program, a bounded LSIP problem need not have optimal solutions. Moreover, the primal and dual problems need not have the same optimal value, as a "positive duality gap" may occur: $v(P)-v(D)>0$. The next two theorems show that the problems in this paper are well-behaved.

Theorem 2.1. $v(D)=v(P)$.

Theorem 2.2. Problems $(P)$ and $(D)$ have optimal solutions.

Hence, the maximum in problem $(D)$ and the minimum in problem $(P)$ are welldefined and they are equal. A nice property of the dual problem is that the space of variables can be restricted without loss of generality to measures with finite support. Proposition 2.1 below establishes the formal result. Let $M_{\mathrm{F}}$ denote the set of finitely supported measures on $Z$. Consider the restricted dual problem, $\left(D_{\mathrm{F}}\right)$, where allocations are defined in $M_{\mathrm{F}} \times M_{\mathrm{F}}$. Denote its optimal value by $v\left(D_{\mathrm{F}}\right)$.

Proposition 2.1. Problem $\left(D_{\mathrm{F}}\right)$ has optimal solutions. Further, $v\left(D_{\mathrm{F}}\right)=v(D)$.

Results of this kind are common to many LSIP programs (Goberna and López [12]).

\footnotetext{
${ }^{7}$ The allocation under autarky (where both types have zero net trade) is feasible and incentive compatible, so problem $(D)$ is consistent. In problem $(P)$, let $\beta_{L}=\beta_{H}=0$ and $q=q^{0}>0$. Since $E U_{i}$ is strictly concave, the right-hand side of both (2.10) and (2.11) is bounded on $Z$. Fixing $\alpha_{L}=\alpha_{L}^{0}$ and $\alpha_{H}=\alpha_{H}^{0}$ sufficiently large ensures that (2.10) and (2.11) hold. By the weak duality theorem (see Krabs [21, Theorem I.3.1]), since problems $(P)$ and $(D)$ are consistent, they are also bounded:

$$
\gamma_{L} E U_{L}(0)+\left(1-\gamma_{L}\right) E U_{H}(0) \leqslant v(D) \leqslant v(P) \leqslant \alpha_{L}^{0}+\alpha_{H}^{0} .
$$
}




\subsection{Full information}

To gain some insight into the linear programming framework, we first consider the case of full information. This case provides a benchmark for the rest of the analysis.

When agent types are public information, a simpler pair of LSIP problems obtains. Theorems 2.2 and 2.1 and Proposition 2.1 extend to these problems. The dual problem $\left(D_{0}\right)$ is obtained by eliminating the incentive constraints in $(D)$. Every first-best allocation is an optimal solution to problem $\left(D_{0}\right)$ for some weight $\gamma_{L} \in(0,1)$. The primal $\left(P_{0}\right)$ is obtained by eliminating the shadow prices of the incentive constraints, $\beta_{L}$ and $\beta_{H}$, and all the associated terms in $(P)$. Thus, the objective function in problem $\left(P_{0}\right)$ is the same as in $(P)$, but the constraint systems are now given by

$$
\alpha_{i} \geqslant v_{i}\left(z_{i} ; q\right) \quad \forall z_{i} \in Z, \quad i=L, H
$$

where

$$
v_{i}\left(z_{i} ; q\right)=\gamma_{i} E U_{i}\left(z_{i}\right)-q \xi_{i} r_{i}\left(z_{i}\right) .
$$

Consider the terms in the function $v_{i}\left(z_{i} ; q\right)$. The first term, $\gamma_{i} E U_{i}\left(z_{i}\right)$, is the contribution to welfare when agents of type $i$ have net trade $z_{i}$. The second term, $q \xi_{i} r_{i}\left(z_{i}\right)$, is the value of the aggregate net trade of these agents when the shadow price of the good is $q$. Thus, $v_{i}\left(z_{i} ; q\right)$ represents the net contribution to social welfare when agents of type $i$ have net trade $z_{i}$ and the shadow price of resources is $q$.

According to (2.13), a feasible value of $\alpha_{i}$ is an upper bound of $v_{i}(\cdot ; q)$ for given $q$. The maximal net contribution of type- $i$ agents at price $q$ is defined as

$$
v_{i}^{*}(q) \equiv \sup _{z_{i} \in Z} v_{i}\left(z_{i} ; q\right)=\sup _{z_{i} \in Z}\left\{\gamma_{i} E U_{i}\left(z_{i}\right)-q \xi_{i} r_{i}\left(z_{i}\right)\right\},
$$

so the primal systems (2.13) can be put in the form

$$
\alpha_{i} \geqslant v_{i}^{*}(q), \quad i=L, H .
$$

Because the objective of problem $\left(P_{0}\right)$ is to minimize the sum of the $\alpha_{i}$ 's, the two constraints in (2.16) bind at an optimum. Thus, the optimal shadow price $q^{*}$ of resources minimizes the sum of the maximal net contributions of the two types: ${ }^{8}$

$$
q^{*}=\arg \left\{\min _{q \geqslant 0}\left\{v_{L}^{*}(q)+v_{H}^{*}(q)\right\}\right\},
$$

and the optimal value of $\alpha_{i}$ gives the maximal net contribution of type $i$ at price $q^{*}$ : $\alpha_{i}^{*}=v_{i}\left(q^{*}\right)$.

First-best allocations. The complementary slackness theorem (see, for instance, Krabs [21, Theorem I.3.3]) allows us to characterize optimal solutions for problems $\left(P_{0}\right)$ and $\left(D_{0}\right)$. According to the theorem, feasible solutions $\left(\alpha_{L}, \alpha_{H}, q\right)$ and $\left(x_{L}, x_{H}\right)$

\footnotetext{
${ }^{8}$ This full information economy is an example of the general problem studied by Makowski and Ostroy [22]. In particular, $\alpha_{i}^{*}(q)$ is the conjugate or indirect utility, redefined in its expected value form for economies with uncertainty. These authors have shown how the fact that the constraints of the primal program (the "pricing problem" in their terminology) can be incorporated into the objective function is characteristic of the LP version of General Equilibrium.
} 
for problems $\left(P_{0}\right)$ and $\left(D_{0}\right)$, respectively, are optimal if and only if they satisfy the complementary slackness conditions:

$$
\begin{aligned}
& q\left(\xi_{L}\left\langle r_{L}, x_{L}\right\rangle+\left(1-\xi_{L}\right)\left\langle r_{H}, x_{H}\right\rangle\right)=0, \\
& \alpha_{i}=v_{i}^{*}(q)=v_{i}\left(z_{i} ; q\right) \quad \text { if } x_{i}\left(z_{i}\right)>0, \quad i=L, H .
\end{aligned}
$$

Condition (2.17) states that the optimal shadow price $q^{*}$ is a complementary multiplier for the resource constraint (2.8). Since the monotonicity of preferences implies that $q^{*}$ is positive, (2.17) implies that the aggregate net trade is zero. Condition (2.18) states that the optimal assignments, $x_{L}^{*}$ and $x_{H}^{*}$, are complementary multiplier vectors for the respective constraint systems in (2.13). This implies that $x_{i}^{*}$ puts weight only on net trades $z_{i}$ that maximize the net contribution to social welfare of type $i$ at price $q^{*}$. However, $v_{i}\left(\cdot ; q^{*}\right)$ is a strictly concave function, and has at most one maximum. Thus, $x_{i}^{*}$ is a degenerate measure; that is, randomization is never optimal. Further, it is easily verified that both types are fully insured as their optimal consumption is independent of the realization of the idiosyncratic shock. In summary, conditions (2.17)-(2.18) yield standard efficiency results for convex economies with full information and no aggregate uncertainty: all agents are fully insured and the aggregate consumption equals the aggregate endowment. We now use a similar characterization to derive the more subtle properties of the optima when agent types are private information.

\subsection{Incentive efficiency}

When types are private information, allocations must provide incentives for the agents to reveal their type. When $\gamma_{L}$ is very large, first-best allocations assign higher consumption to type $L$ than to type $H$, so agents of type $H$ are inclined to lie. Similarly, when $\gamma_{L}$ is very low, first-best allocations give higher consumption to type $H$, which induces the agents of type $L$ to lie. It is easily verified that, for some intermediate weight, it is optimal that both types consume the ex-post average endowment $\bar{w}$ with certainty. This weight is given by

$$
\bar{\gamma}_{L}=\left(1+\frac{\left(1-\xi_{L}\right) U_{L}^{\prime}(\bar{\omega})}{\xi_{L} U_{H}^{\prime}(\bar{\omega})}\right)^{-1},
$$

and corresponds to the only first-best allocation that is also incentive compatible. The next proposition describes a partitioning of the set of incentive efficient allocations into three regions according to which incentive compatibility constraint binds.

Proposition 2.2. The set of incentive efficient allocations has three regions:

(i) When $\gamma_{L}=\bar{\gamma}_{L}$, the incentive efficient allocation assigns each type $\bar{\omega}$ units of consumption in every state, and the two incentive constraints trivially bind. Thus, $\beta_{L}=\beta_{H}=0$. 
(ii) When $\bar{\gamma}_{L}<\gamma_{L}<1$, incentive efficient allocations assign higher expected consumption to type $L$ than to type $H$ and, only the incentive constraint of type $H$ binds. In this case, $\beta_{L}=0$ and $\beta_{H}>0$.

(iii) When $0<\gamma_{L}<\bar{\gamma}_{L}$, incentive efficient allocations assign higher expected consumption to type $H$ than to type $L$ and, only the incentive constraint of type $L$ binds. In this case, $\beta_{L}>0$ and $\beta_{H}=0$.

Cases (ii) and (iii) in Proposition 2.2 are essentially symmetric and can be studied separately. ${ }^{9}$

\subsubsection{The incentives of type- $H$ agents}

In this section, we characterize incentive efficient allocations in which only the incentive constraint of type $H$ binds. Throughout we let $\gamma_{L} \in\left(\bar{\gamma}_{L}, 1\right)$ so, by Proposition 2.2, $\beta_{L}=0$. The first constraint system in problem $(P)$ is then given by

$$
\alpha_{L} \geqslant v_{L}\left(z_{L} ; \beta_{H}, q\right) \quad \forall z_{L} \in Z,
$$

where the function

$$
v_{L}\left(z_{L} ; \beta_{H}, q\right)=\gamma_{L} E U_{L}\left(z_{L}\right)-q \xi_{L} r_{L}\left(z_{L}\right)-\beta_{H} E U_{H}\left(z_{L}\right)
$$

represents the net contribution to social welfare of type-L agents when types are private information. The net contribution of type $L$ is adjusted with respect to its full information version and, unlike the latter, depends on the shadow price of the incentive constraint of type $H$. Specifically, a new term arises which is not present under full information: $-\beta_{H} E U_{H}\left(z_{L}\right)$. This term reflects an external cost that arises as a result of the effect of the assignments to type $L$ on type- $H$ agents. That is, the better the assignment of type $L$ in the eyes of type- $H$ agents, the more costly it is to prevent the latter from lying. The total cost of assignments to type $L$ is given by the sum of the resource cost and the external cost: $q \xi_{L} r_{L}\left(z_{L}\right)+\beta_{H} E U_{H}\left(z_{L}\right)$. When $\beta_{H}$ is positive, the external cost is positive and, for a given $q$, the total shadow cost is higher than under full information. Note that, since $E U_{H}(\cdot)$ is strictly concave, the total cost is not a convex function of $z_{L}$.

The second constraint system is given by

$$
\alpha_{H} \geqslant v_{H}\left(z_{H} ; \beta_{H}, q\right) \quad \forall z_{H} \in Z,
$$

where the function

$$
v_{H}\left(z_{H} ; \beta_{H}, q\right)=\left(1-\gamma_{L}\right) E U_{H}\left(z_{H}\right)-q\left(1-\xi_{L}\right) r_{H}\left(z_{H}\right)+\beta_{H} E U_{H}\left(z_{H}\right)
$$

represents the net contribution to social welfare of type- $H$ agents at prices $\beta_{H}$ and $q$. The third term in the function reflects a benefit of assignments to type $H$ on the incentives of these agents. Clearly, the higher the utility that type- $H$ agents derive from their own assignment, the more incentives they have to report the truth. The shadow cost of assignments to type $H$ is given by the resource cost net of the benefit

\footnotetext{
${ }^{9}$ Prescott and Townsend [26, Section 3] obtain a complete specification of the set of incentive efficient allocations when utilities are identical across types using the first-order conditions of the planner problem. In this case, $\bar{\gamma}_{L}=\bar{\xi}_{L}$.
} 
on incentives: $q\left(1-\xi_{L}\right) r_{H}\left(z_{H}\right)-\beta_{H} E U_{H}\left(z_{H}\right)$. When $\beta_{H}$ is positive, the benefit on incentives is positive and, for a given $q$, the total cost is lower than under full information. In this case, the cost is a convex function of $z_{H}$.

According to (2.19) and (2.21), a feasible value of $\alpha_{i}$ is an upper bound of $v_{i}\left(\cdot ; \beta_{H}, q\right)$ for given $\beta_{H}$ and $q$. The maximal net social contribution of type- $i$ agents at prices $\beta_{H}$ and $q$ is defined as

$$
v_{i}^{*}\left(\beta_{H}, q\right) \equiv \sup _{z_{i} \in Z} v_{i}\left(z_{i} ; \beta_{H}, q\right) .
$$

Constraints (2.19) and (2.21) can then be expressed as

$$
\alpha_{i} \geqslant v_{i}^{*}\left(\beta_{H}, q\right), \quad i=L, H .
$$

Thus, the optimal prices $\beta_{H}^{*}$ and $q^{*}$ minimize the sum of the maximal net contributions of the two types:

$$
\left(q^{*}, \beta_{H}^{*}\right) \in \arg \left\{\min _{\beta_{H}, q \geqslant 0}\left\{v_{L}^{*}\left(\beta_{H}, q\right)+v_{H}^{*}\left(\beta_{H}, q\right)\right\}\right\},
$$

and the optimal net contributions are given by $\alpha_{i}^{*}=v_{i}^{*}\left(\beta_{H}^{*}, q^{*}\right)$ for $i=L, H$.

The principal result in this section is the characterization of incentive efficient allocations. According to the complementary slackness theorem, feasible solutions $\left(\alpha_{L}, \alpha_{H}, \beta_{H}, q\right)$ and $\left(x_{L}, x_{H}\right)$ for problems $(P)$ and $(D)$, respectively, are optimal if and only if:

$$
\begin{aligned}
& \beta_{H}\left(\left\langle E U_{H}, x_{H}\right\rangle-\left\langle E U_{H}, x_{L}\right\rangle\right)=0, \\
& q\left(\xi_{L}\left\langle r_{L}, x_{L}\right\rangle+\left(1-\xi_{L}\right)\left\langle r_{H}, x_{H}\right\rangle\right)=0, \\
& \alpha_{i}=v_{i}^{*}\left(\beta_{H}, q\right)=v_{i}\left(z_{i} ; \beta_{H}, q\right) \quad \text { if } x_{i}\left(z_{i}\right)>0, i=L, H .
\end{aligned}
$$

By Proposition 2.2 we know that $\beta_{H}^{*}>0$, and it can be verified that $q^{*}>0 .{ }^{10}$ Thus, incentive efficient allocations satisfy the following three properties. First, an agent of type $H$ is indifferent between her assignment and that of type $L$. Second, the aggregate net trade is zero. Third, the lottery $x_{i}^{*}$ assigned to type $i$ puts weight only on net trades that maximize the net contribution of type $i$ at prices $q^{*}$ and $\beta_{H}^{*}$. The third property leads to the following result which stems directly from the strict concavity of $v_{H}\left(\cdot ; \beta_{H}^{*}, q^{*}\right)$ and first-order conditions.

Proposition 2.3. $x_{H}^{*}$ is degenerate and provides full insurance.

Proposition 2.3 states that agents of type $H$ should be fully insured when their average consumption is below $\bar{\omega}$. Full insurance increases both the utility of type $H$ for a given resource cost and her incentives to report the truth (decreasing the total cost of the assignment). Since the incentive constraint of type $L$ is not binding, type $L$ wants to tell the truth. However, more insurance to type $L$, while increasing her own

\footnotetext{
${ }^{10}$ If $q^{*}=0$ and $U_{H}$ is unbounded then $v_{H}\left(\cdot ; \beta^{*}, q^{*}\right)$ is unbounded on $Z$, which contradicts (2.27). If $U_{H}$ is bounded, then $v_{H}\left(\cdot ; \beta^{*}, 0\right)$ does not have a maximum (recall that $\lim _{c \rightarrow \infty} U_{i}^{\prime}(c)=0$ ), a contradiction since $(D)$ is solvable and the support of $x_{H}^{*}$ is non-empty.
} 
utility, may raise the incentives of type $H$ to lie. Then, for a given resource cost, an increase in insurance to type $L$ raises the total cost. As a result, the optimal assignment to type $L$ is distorted from full insurance. Since the total cost is not convex on $z_{L}$, optimal assignments may even be random. If utilities are typeinvariant (as in Rothschild and Stiglitz [28] and Wilson [30]), however, optimal assignments to type $L$ are deterministic. ${ }^{11}$

Proposition 2.4. If utilities are type-invariant, then $x_{L}^{*}$ is degenerate and assigns lower consumption in state 1 than in state 2.

Given the consumption level of type $H$, the planner chooses $x_{L}$ so as to increase the utility of type $L$ as much as possible without inducing type $H$ to lie. For a given resource cost, the planner may increase the net social contribution of type $L$ (i.e., widen the gap between the utility of type $L$ and the external cost of the assignment) by exploiting differences in the preferences of the two types. Because type $L$ is more likely to be in state 2 than type $H$, assignments which give higher consumption in state 2 relative to state 1 are relatively more attractive to type $L$ than to type $H$. This explains why partial insurance to type $L$ is incentive efficient. Lottery assignments, in turn, exploit differences in preferences for risk. When utilities are identical across types, there are no such differences and lotteries do not help enhance efficiency. There are economies, however, where lotteries play a useful role. Consider the extreme case where agents of type $L$ are risk neutral and agents of type $H$ are risk averse. One can then easily devise a random allocation which is incentive compatible and first best efficient. First, agents announcing type $H$ are assigned their first-best deterministic consumption level. Agents announcing type $L$, on the other hand, receive a non-degenerate lottery. Whereas the implied expected consumption (and, hence, the utility) of type $L$ is also the first-best one, the risk involved is such that the certainty equivalent that type- $H$ agents assign to the lottery is no greater than their own deterministic consumption. This prevents any misrepresentation.

The next proposition shows that, when type $L$ is at least as risk averse as type $H$, lotteries are not useful. Let $A_{i}: \mathbb{R}_{+} \rightarrow \mathbb{R}_{+}$denote the index of absolute risk aversion for type $i$; that is, $A_{i}(c)=-\frac{U_{i}^{\prime \prime}(c)}{U_{i}^{\prime}(c)}$ for $c \in \mathbb{R}_{+}$.

Proposition 2.5. If $A_{L}(c) \geqslant A_{H}(c)$ for all $c \in \mathbf{R}_{+}$, then $x_{L}^{*}$ is degenerate and assigns lower consumption in state 1 than in state 2.

\subsubsection{The incentives of type- $L$ agents}

An analysis analogous to the one in the previous section allows us to characterize incentive efficient allocations in which the incentive constraint of type $L$ binds. In this case, incentive efficient allocations provide full insurance to type $L$ and over

\footnotetext{
${ }^{11}$ The same result is obtained by Prescott and Townsend [26] who derive also the result in Proposition 2.3 for an environment with type-invariant utilities.
} 
insurance to type $H$. Full insurance to a type- $L$ agent increases her utility as well as her incentive to tell the truth. The optimal way to induce type $L$ to tell the truth is to provide over insurance to type $H$. Intuitively, an over insured position is less attractive to type- $L$ agents than to type- $H$ agents since the former are less likely to be in the low endowment state. If type $L$ is sufficiently more risk averse than type $H$, the assignment to type $H$ may be random, since type $L$ is more reluctant to accept random assignments than type $H$. By contrast, if type $H$ is at least as risk averse as type $L$, optimal assignments to type $H$ are deterministic. The next proposition summarizes the properties of incentive efficient allocations when the incentive constraint of type $L$ binds.

Proposition 2.6. For any $\gamma_{L} \in\left(0, \bar{\gamma}_{L}\right)$, incentive efficient allocations satisfy the following.

(i) $x_{L}^{*}$ is degenerate and provides full insurance; and

(ii) If utilities are type-invariant, then $x_{H}^{*}$ is degenerate and assigns higher consumption in state 1 than in state 2. The same result holds if utilities are not type invariant, and $A_{H}(c) \geqslant A_{L}(c)$ for all $c \in \mathbb{R}_{+}$.

\section{Moral hazard}

\subsection{The economy}

Consider an exchange economy with two goods, namely leisure time and a single consumption good, and a measure one of ex ante identical agents. Each agent faces an idiosyncratic endowment shock leading to two possible states, $s=1,2$. In state $s$, the agent is endowed with $\omega_{s}$ units of consumption where $0<\omega_{1}<\omega_{2}$. Prior to the realization of the shock, the agent is endowed with one unit of time which he allocates between leisure and effort in preventing the realization of state 1 . The agent can exert either high or low effort, with the set of effort levels denoted by $E=$ $\left\{e_{L}, e_{H}\right\}$, where $0<e_{L}<e_{H}<1$. Exerting high rather than low effort reduces the probability that the agent will end up in state 1 . The probability of state 1 with high and low effort is denoted by $\theta_{H}$ and $\theta_{L}$, respectively, so that $0<\theta_{H}<\theta_{L}<1$. Agents have von Neumann-Morgenstern preferences as defined by the utility function $u: E \times \mathbb{R}_{+} \rightarrow \mathbb{R}$. The utility of consumption $c$ under effort $e_{i}$ is given by $U_{i}(c)=$ $u\left(e_{i}, c\right)$, where $U_{i}$ is assumed twice continuously differentiable, strictly increasing, and strictly concave with $\lim _{c \rightarrow 0} U_{i}^{\prime}(c)=\infty$ and $\lim _{c \rightarrow \infty} U_{i}^{\prime}(c)=0$. Since effort is costly, we assume that there is some positive constant $d$ such that $U_{L}(c)-U_{H}(c)>d$ for all $c \in \mathbb{R}_{+}$.

Idiosyncratic shocks are independent and render no aggregate uncertainty. The expost average endowment of the agents who provide effort $e_{i}$ is then given by $\bar{\omega}_{i}=$ $\theta_{i} \omega_{1}+\left(1-\theta_{i}\right) \omega_{2}$. The structure of uncertainty is common knowledge and the realization of the endowment shocks is observable. State-contingent net trades are perfectly verifiable and fully enforceable. However, effort is private information. 


\subsection{Allocations}

In this section, we define feasible and incentive compatible allocations. Then we define incentive efficient allocations.

Let $Z \subset \mathbb{R}_{+}^{2}$ denote the net trade set of an agent. For any $z=\left(z_{1}, z_{2}\right) \in Z$, the expected net trade of an agent with effort $e_{i}$ is

$$
r_{i}\left(z_{1}, z_{2}\right)=\theta_{i} z_{1}+\left(1-\theta_{i}\right) z_{2}
$$

and his expected utility is given by

$$
E U_{i}\left(z_{1}, z_{2}\right)=\theta_{i} U_{i}\left(\omega_{1}+z_{1}\right)+\left(1-\theta_{i}\right) U_{i}\left(\omega_{2}+z_{2}\right) .
$$

An allocation in this economy specifies an effort level and a net trade for each agent. Both specifications are allowed to be random and are given as follows. First, the agent receives a lottery which prescribes an effort level. After the agent chooses his effort and conditional on the prescription received, a second lottery specifies a net trade. It is useful for our purposes to describe an allocation as a pair of measures $\left(x_{L}, x_{H}\right) \in M_{\mathrm{c}}(Z) \times M_{\mathrm{c}}(Z)$ such that

$$
\left\langle\mathscr{I}, x_{L}+x_{H}\right\rangle=1, \quad x_{i} \geqslant 0, \quad i=L, H .^{12}
$$

Here, $\left\|x_{i}\right\|=\left\langle\mathscr{I}, x_{i}\right\rangle$ is the probability that effort $e_{i}$ is specified in the first lottery, and the equality in (3.28) is an adding-up condition. ${ }^{13}$ In addition, $\frac{1}{\left\|x_{i}\right\|} x_{i}$ is a probability measure which describes the random net trade assigned conditional on specification $e_{i}$ (i.e., the second lottery). Note that the uncertainty involved in an allocation is resolved in two steps. In the first step, the agent may be uncertain about the effort that he will be asked to provide. This occurs when both $\left\|x_{L}\right\|$ and $\left\|x_{H}\right\|$ are positive. In the second step, the agent finds out his effort specification, but he may be uncertain about his contingent consumption plan. This occurs when $\frac{1}{\left\|x_{i}\right\|} x_{i}$ is a nondegenerate measure. Allowing for random effort is natural since the consumption set, $E \times \mathbb{R}_{+}$, displays indivisibilities. As we shall see, the role for random net trade assignments arises from the unobservability of effort.

From the perspective of the entire economy, $\left\|x_{i}\right\|$ is the fraction of agents who are assigned $e_{i}$, and $\frac{1}{\left\|x_{i}\right\|} x_{i}$ is the distribution of their net trades. The ex-post aggregate net trade of these agents is given by $\left\langle r_{i}, x_{i}\right\rangle=\int_{Z} r_{i} d x_{i}$ (provided the agents conform to their specification). An allocation is feasible if the aggregate net trade is non-positive:

$$
\left\langle r_{L}, x_{L}\right\rangle+\left\langle r_{H}, x_{H}\right\rangle \leqslant 0 .
$$

When effort $e_{i}$ is specified but $e_{j}$ is the actual effort provided, the agent's expected utility is $\frac{1}{\left\|x_{i}\right\|}\left\langle E U_{j}, x_{i}\right\rangle$. An allocation is incentive compatible if it is not in the interest

\footnotetext{
${ }^{12}$ See Prescott and Townsend [26] and Bennardo and Chiappori [3] for equivalent descriptions of the space of allocations.

${ }^{13}$ Here, $\left\|x_{i}\right\|=\left\langle\mathscr{I}, x_{i}\right\rangle=x_{i}(Z)$ is the total variation of $x_{i}$.
} 
of the agents to deviate from their specifications:

$$
\left\langle E U_{i}, x_{i}\right\rangle \geqslant\left\langle E U_{j}, x_{i}\right\rangle, \quad j \neq i, \quad i=L, H .
$$

An incentive efficient allocation is a feasible and incentive compatible allocation that maximizes the ex-ante expected utility of the agents.

\subsection{The primal and dual problems}

An incentive efficient allocation can be determined as a solution to a planning problem, more precisely a dual LSIP problem. The problem is to choose an allocation $\left(x_{L}, x_{H}\right) \in M_{\mathrm{c}}(Z) \times M_{\mathrm{c}}(Z)$ that solves

$$
\begin{aligned}
& \text { (D) } \sup \left\langle E U_{L}, x_{L}\right\rangle+\left\langle E U_{H}, x_{H}\right\rangle \text { s.t. } \\
& \left\langle\mathscr{I}, x_{L}+x_{H}\right\rangle=1 \text {, } \\
& -\left\langle E U_{L}, x_{L}\right\rangle+\left\langle E U_{H}, x_{L}\right\rangle \leqslant 0, \\
& \left\langle E U_{L}, x_{H}\right\rangle-\left\langle E U_{H}, x_{H}\right\rangle \leqslant 0, \\
& \left\langle r_{L}, x_{L}\right\rangle+\left\langle r_{H}, x_{H}\right\rangle \leqslant 0, \\
& x_{L}, x_{H} \geqslant 0 \text {. }
\end{aligned}
$$

The primal LSIP problem consists of finding a quadruple $\left(\alpha, \beta_{L}, \beta_{H}, q\right) \in \mathbb{R}^{4}$ that solves

$(P) \inf \alpha$

s.t.

$$
\begin{aligned}
& \alpha \geqslant E U_{L}(z)-\beta_{L}\left[E U_{H}(z)-E U_{L}(z)\right]-q r_{L}(z) \quad \forall z \in Z, \\
& \alpha \geqslant E U_{H}(z)-\beta_{H}\left[E U_{L}(z)-E U_{H}(z)\right]-q r_{H}(z) \quad \forall z \in Z, \\
& \beta_{L}, \beta_{H}, q \geqslant 0,
\end{aligned}
$$

where $\alpha,\left(\beta_{L}, \beta_{H}\right)$, and $q$ are the shadow prices of the adding-up constraint (3.31), the incentive constraints (3.32)-(3.33), and the resource constraint (3.34), respectively. In Appendix A, we show that problems $(P)$ and $(D)$ have optimal solutions and that their optimal values coincide. We also show that, in characterizing incentive efficient allocations, there is no loss of generality in restricting attention to measures with finite support. These results are analogous to Theorems 2.2 and 2.1 and Proposition 2.1 in Section 2.3.

\subsection{Incentive efficiency}

In this section, we characterize incentive efficient allocations. Since the aggregate endowment is constant, under full information it is optimal that each agent consumes with certainty the average endowment in the economy. However, since 
effort is costly and cannot be publicly observed, an agent who is fully insured will shirk to low effort when high effort is specified. For this reason, allocations which specify high effort with positive probability can only provide partial insurance. On the other hand, agents must be subject to the minimum risk possible that is compatible with their incentives to conform to a high-effort specification. So the incentive constraint (3.33) binds with $\beta_{H}>0$. Implementing a low-effort specification is trivial. Since the incentive constraint (3.32) does not bind, we may let $\beta_{L}=0$.

The first constraint system in problem $(P)$ is given by

$$
\alpha \geqslant v_{L}\left(z_{L} ; q\right) \quad \forall z_{L} \in Z,
$$

where the function

$$
v_{L}\left(z_{L} ; q\right)=E U_{L}\left(z_{L}\right)-q r_{L}\left(z_{L}\right)
$$

represents the net contribution to social welfare with low effort. The first term in (3.40) is the contribution to welfare when agents are assigned effort $e_{L}$ and net trade $z_{L}$. The second term is the value of the associated aggregate net trade when the shadow price of the good is $q$. Note that there are no welfare effects of incentives, so the net social contribution with low effort is the same both under full and under private information.

The second constraint system is given by

$$
\alpha \geqslant v_{H}\left(z_{H} ; \beta_{H}, q\right) \quad \forall z_{H} \in Z,
$$

where the function

$$
v_{H}\left(z_{H} ; \beta_{H}, q\right)=E U_{H}\left(z_{H}\right)-q r_{H}\left(z_{H}\right)-\beta_{H}\left[E U_{L}\left(z_{H}\right)-E U_{H}\left(z_{H}\right)\right]
$$

represents the net contribution to social welfare with high effort. The first and second terms in (3.42) are the contribution to welfare when agents are assigned effort $e_{H}$ and net trade $z_{H}$, and the value of the associated aggregate net trade, respectively. The third term reflects an additional welfare effect which is associated with the incentives of the agents to provide high effort. If the net trade is such that the agents have incentives to deviate to $e_{L}$, the term is negative and reflects a cost which is proportional to the utility gain in the deviation. If the net trade is such that the agents want to conform to $e_{H}$, the term is positive and reflects a benefit which is proportional to the utility loss that a deviation would imply. The direct (i.e. full information) net contribution of the assignment is thus adjusted upward (downward) when it gives the right (wrong) incentives. The total cost of an assignment under high effort is given by the resource cost net of the welfare effect of incentives: $q r_{H}\left(z_{H}\right)+$ $\beta_{H}\left[E U_{L}\left(z_{H}\right)-E U_{H}\left(z_{H}\right)\right]$. Note that the cost function is not convex.

The maximal net contributions with high and low effort at prices $\beta_{H}$ and $q$ are:

$$
\begin{aligned}
& v_{L}^{*}(q) \equiv \sup _{z_{L} \in Z} v_{L}\left(z_{L} ; q\right), \\
& v_{H}^{*}\left(\beta_{H}, q\right) \equiv \sup _{z_{H} \in Z} v_{H}\left(z_{H} ; \beta_{H}, q\right) .
\end{aligned}
$$


It is thus possible to write conditions (3.39) and (3.41) as

$$
\alpha \geqslant v^{*}\left(\beta_{H}, q\right),
$$

where $v^{*}\left(\beta_{H}, q\right)$ is the largest of the two maximal net contributions:

$$
v^{*}\left(\beta_{H}, q\right) \equiv \max \left\{v_{L}^{*}(q), v_{H}^{*}\left(\beta_{H}, q\right)\right\} .
$$

Because the objective of the primal problem is to minimize $\alpha$, the optimal prices $\beta_{H}^{*}$ and $q^{*}$ are determined by minimizing $v^{*}\left(\beta_{H}, q\right)$ :

$$
\left(\beta_{H}^{*}, q^{*}\right) \in \arg \left\{\min _{\beta_{H}, q \geqslant 0}\left\{v^{*}\left(\beta_{H}, q\right)\right\}\right\},
$$

while optimal net contribution is given by $\alpha^{*}=v^{*}\left(\beta_{H}^{*}, q^{*}\right)$.

We now turn to the characterization of incentive efficient allocations. By the complementary slackness theorem, if $\left(\alpha, \beta_{H}, q\right)$ and $\left(x_{L}, x_{H}\right)$ are feasible for problems $(P)$ and $(D)$, respectively, then they are optimal if and only if:

$$
\begin{aligned}
& \beta_{H}\left(\left\langle E U_{L}-E U_{H}, x_{H}\right\rangle\right)=0, \\
& q\left(\left\langle r_{L}, x_{L}\right\rangle+\left\langle r_{H}, x_{H}\right\rangle\right)=0, \\
& \alpha=v^{*}\left(\beta_{H}, q\right)=v_{i}\left(z_{i} ; \beta_{H}, q\right) \text { if } x_{i}\left(z_{i}\right)>0, i=L, H .
\end{aligned}
$$

We have already noted that $\beta_{H}^{*}>0$, and it can be checked that $q^{*}>0$. Thus, an incentive efficient allocation $\left(x_{L}^{*}, x_{H}^{*}\right)$ has the following three properties. First, when $e_{H}$ is assigned, the agent is indifferent between exerting effort $\left(e_{H}\right)$ and shirking $\left(e_{L}\right)$. Second, the aggregate net trade is zero. Third, $x_{i}^{*}$ puts weight only on net trades which achieve the optimal net contribution $\alpha^{*}$. That is, $x_{L}^{*}\left(z_{L}\right)$ is positive provided: (i) $z_{L}$ maximizes $v_{L}\left(\cdot ; q^{*}\right)$, and (ii) $v^{*}\left(\beta_{H}^{*}, q^{*}\right)=v_{L}\left(q^{*}\right)$. Similarly, $x_{H}^{*}\left(z_{H}\right)$ is positive provided: (i) $z_{H}$ maximizes $v_{H}\left(\cdot ; \beta_{H}^{*}, q^{*}\right)$, and (ii) $v^{*}\left(\beta_{H}^{*}, q^{*}\right)=v_{H}\left(\beta_{H}^{*}, q^{*}\right)$. The next proposition follows from the third property and is direct from the strict concavity of $v_{L}\left(\cdot ; q^{*}\right)$ and first-order conditions.

Proposition 3.1. If $\left\|x_{L}^{*}\right\|>0$ then $x_{L}^{*}$ is degenerate and provides full insurance.

Since a low effort assignment does not generate incentive effects, an agent who is assigned $e_{L}$ should be fully insured. Under a high effort specification, however, an increase in insurance may raise the incentives to shirk (increasing the shadow cost of the assignment). Since the shadow cost function is not convex, net trade assignments under high effort may be random. When utility is separable in effort and consumption, however, optimal assignments under high effort are deterministic.

Proposition 3.2. Suppose that utility is separable in consumption and effort. If $\left\|x_{H}^{*}\right\|>0$ then $x_{H}^{*}$ is degenerate and assigns lower consumption in state 1 than in state 2.

The planner would like to increase as much as possible the utility of agents who are specified high effort without adversely affecting their incentives. The planner may 
increase the net contribution with high effort by exploiting differences in preferences with high and low effort. Partial insurance makes high effort relatively more attractive because it raises the probability of being in the high consumption state. Random net trade assignments exploit differences in preferences for risk. When utility is separable, there are not such differences and optimal assignments are deterministic. Consider, however, the extreme case where an agent is risk neutral when his effort is high and risk averse when it is low. Then it is easy to find a random allocation which is incentive compatible and first-best efficient. In this allocation, agents who are specified high effort are assigned a random net trade which yields the first-best expected consumption and involves sufficient risk for the agents not to have incentives to shirk to low effort.

The next proposition shows that, if risk aversion does not decrease with effort, random net trade assignments are not optimal. ${ }^{14}$ Let $A_{i}: \mathbb{R}_{+} \rightarrow \mathbb{R}_{+}$be the index of absolute risk aversion of an agent with effort $e_{i}$; that is, $A_{i}(c)=-\frac{U_{i}^{\prime \prime}(c)}{U_{i}^{\prime}(c)}$ for $c \in \mathbb{R}_{+}$.

Proposition 3.3. Suppose that $A_{H}(c) \geqslant A_{L}(c)$ for all $c \in \mathbb{R}_{+}$. If $\left\|x_{H}^{*}\right\|>0$ then $x_{H}^{*}$ is degenerate and assigns lower consumption in state 1 than in state 2.

Remark. The conditions which characterize incentive efficient allocations with adverse selection and moral hazard are very similar (conditions (2.25)-(2.27) and (3.46)-(3.48), respectively). The main differences between them relate to the definition of the net contribution functions in each model, and thereby in the third condition of the characterization. The net contributions differ in the terms that describe the welfare effects of incentives. With adverse selection, the assignments of both types generate incentive effects. In particular, the final term in the net contribution of the type with the highest average consumption reflects an external cost because the assignment of this type affects the truth-telling incentives of the other type. With moral hazard, only the assignment of those agents who are specified high effort generates incentive effects. The main difference compared with the adverse selection model is that the final term in the net contribution with high effort does not reflect an external cost. With moral hazard, it is the incentives of the agents who receive the high-effort specification (not the incentives of others) that are affected. ${ }^{15}$ Another difference is that, while in the adverse selection model the fraction of each type is exogenously given, in the moral hazard model the fraction of agents assigned to each effort level is endogenous. For this reason in the first model the net contributions of the two types are studied independently, while in the second model the net contributions with high and low effort must be compared in order to determine the optimal fractions of agents assigned to each effort level. Assuming that it is never optimal that all agents provide low effort, the fraction of agents assigned

\footnotetext{
${ }^{14}$ Arnott and Stiglitz [2] derive this result through a different argument.

${ }^{15}$ The source of this difference is the different form of the incentive constraints in the two models. Whereas with adverse selection both incentive constraints depend on assignments of the two types $x_{L}$ and $x_{H}$, with moral hazard the (relevant) incentive constraint depends only on the net trade assignment under high effort $x_{H}$.
} 
to high effort is one if the maximal contribution with high effort is higher than the maximal contribution with low effort at the optimal prices, and is a number between zero and one if the two maximal contributions are equal.

The remainder of this section focuses on optimal effort assignments. To avoid trivial solutions, we assume throughout that it is not incentive efficient to assign low effort with probability one and to provide the agents with full insurance. ${ }^{16}$ Below we give conditions under which the optimal effort is random.

Consider the most preferred allocation which is feasible and incentive compatible, and specifies high effort with probability one. There may exist a feasible and incentive compatible allocation which specifies low effort with positive probability and is strictly preferred by the agents. When a fraction of the population provides low effort, the aggregate endowment decreases. Yet, if this fraction is taken to be small, the loss in endowment is also small. Starting from the original high effort allocation, it is then feasible to give an arbitrarily high fixed consumption to a sufficiently small fraction of agents and allow these agents to provide low effort without decreasing the average consumption of others by too much. The cost of introducing this lottery is that, due to a reduction in expected consumption, agents are slightly worse off if high effort is specified. The benefit is that, ex ante, every agent has a positive probability of being fully insured, exerting less effort and receiving a highly subsidized consumption level. ${ }^{17}$ Since marginal utility of consumption decreases to zero, the cost of giving up a small amount of expected consumption becomes negligible as agents get wealthier. The disutility of effort, however, is strictly positive. Proposition 3.4 asserts that, under the natural assumption that marginal utility of consumption does not increase with effort (decrease with leisure) and as long as the loss in expected endowment from switching from high to low effort, $\bar{\omega}_{H}-\bar{\omega}_{L}$, is bounded, agents are willing to give up some expected consumption to participate in the lottery provided they are sufficiently wealthy.

\section{Proposition 3.4. Suppose that}

(i) $U_{H}^{\prime}(c) \leqslant U_{L}^{\prime}(c)$ for all $c \in \mathbb{R}_{+}$, and

(ii) $\left(\theta_{L}-\theta_{H}\right)\left(\omega_{2}-\omega_{1}\right)<M$ for some constant $M$, so $\left(\bar{\omega}_{H}-\bar{\omega}_{L}\right)$ is bounded.

Then there exists a threshold $\hat{\omega}_{H}$ such that, if $\bar{\omega}_{H} \geqslant \hat{\omega}_{H}$ then $\left\|x_{H}^{*}\right\|<1$.

In recent work, Bennardo and Chiappori [3] study a moral hazard environment where the optimal effort is typically random. ${ }^{18}$ In their model, consumption and leisure are complementary goods, so the marginal utility of consumption decreases with effort, and the cost of effort increases with consumption. A key result in that

\footnotetext{
${ }^{16}$ Assume there exists a feasible and incentive compatible allocation $\left(x_{L}, x_{H}\right)$ such that $x_{L}=0$ and $\left\langle E U_{H}, x_{H}\right\rangle>U_{L}\left(\bar{\omega}_{L}\right)$.

${ }^{17}$ That is, of "winning the lottery" and reducing effort.

${ }^{18}$ Most models in the partial equilibrium literature consider only deterministic effort prescriptions. See, however, the general equilibrium model of Prescott and Townsend [26].
} 
paper is that, if the marginal utility of consumption decreases fast enough with effort then there is a limit to the amount of expected consumption that agents can receive while still being willing to provide high effort. Put differently, a really wealthy individual may have no incentive to provide high effort. When the aggregate endowment exceeds a threshold level, part of the available resources cannot be consumed if high effort is specified with probability one. In this case, the best higheffort allocation cannot be incentive efficient because part of the aggregate endowment is not consumed. Proposition 3.5 is a similar, slightly stronger result than the result in Bennardo and Chiappori [3, Lemma 3.6]. It shows that the key assumption behind the Bennardo-Chiappori result is that marginal utility of consumption ought to decrease faster with high than with low effort (condition (ii)).

\section{Proposition 3.5. Suppose that}

(i) $U_{H}^{\prime}(c) \leqslant U_{L}^{\prime}(c)$ for all $c \in \mathbb{R}_{+}$, and

(ii) $\lim _{c \rightarrow \infty} \frac{U_{H}^{\prime}(c)}{U_{L}^{\prime}(c)}=0$.

Then there exists a threshold $\check{\omega}_{H}$ such that, if $\bar{\omega}_{H} \geqslant \check{\omega}_{H}$ then any allocation with $\left\|x_{H}\right\|=1$ satisfies the resource constraint (3.34) with strict inequality. In this case, $\left\|x_{H}^{*}\right\|<1$.

Condition (ii) in Proposition 3.5 can replace condition (ii) in Proposition 3.4 to yield the same result on the optimality of randomization over effort. The new condition implies that, with a sufficiently high aggregate endowment, there are resources available for free. One can use these free resources to give an arbitrarily high fixed consumption to a sufficiently small fraction of agents (chosen at random) and allow them to provide low effort. Hence, randomization over effort increases the ex ante expected utility of the agents. In turn, condition (ii) in Proposition 3.4 is necessary to ensure that the cost of the resources that are transferred to the agents who provide low effort is small when these agents are a small fraction of the population (the loss in endowment when an agent provides low rather than high effort is bounded). Another difference between Propositions 3.4 and 3.5 is that random effort prescriptions may be optimal in economies where agents have relatively low endowments provided that the ratio $\frac{U_{H}^{\prime}(c)}{U_{L}^{\prime}(c)}$ goes to zero sufficiently fast.

\section{Conclusion}

Using the techniques of linear programming and duality theory, we have characterized incentive efficient allocations in two standard environments with asymmetric information. The decentralization of incentive efficient allocations as competitive equilibria is an important issue that remains to be pursued, in particular for the case of adverse selection. We conclude with a brief discussion of the possible implications of the paper's finding for the study of this important problem and of the relationship with the literature on decentralization. 
In their seminal work, Prescott and Townsend [26,27] show that competitive equilibria exist and are incentive efficient if agents trade before the asymmetry of information is realized (e.g. moral hazard). Their approach, however, does not extend to economies where trade takes place after the asymmetric information is realized (e.g. adverse selection). Our work brings to light an important difference between adverse selection and moral hazard. With adverse selection the welfare effects that arise from incentive constraints on the allocations reflect external effects, while with moral hazard they do not. We conjecture that this key difference may be driving the results of Prescott and Townsend [26]. In a recent paper, Bisin and Gottardi [5] study a similar adverse selection model where they identify a consumption externality. They argue that, since the agents' consumption plans must satisfy incentive constraints, the consumption plan of each agent type affects the set of admissible plans of the other type. An enlarged set of markets which includes markets for consumption rights is then described for which competitive equilibria exist and appropriate versions of the first and second welfare theorems hold. However, if markets for consumption rights are not available, equilibria exist but need not be incentive efficient. The importance of external effects in economies with asymmetric information is highlighted also by Greenwald and Stiglitz [13] and Arnott et al. [1].

In recent work, Bennardo and Chiappori [3] study a moral hazard model with both idiosyncratic and aggregate risk where they show that competitive equilibria exist and are incentive efficient. Further, if consumption and leisure are complements and the cost of effort increases fast enough with consumption, the optimal effort is random. In this paper we have derived a similar, slightly stronger result for the case of purely idiosyncratic uncertainty and have shown that the optimal effort is random also if the aggregate endowment is sufficiently large.

We believe that linear programming is a promising route for studying the decentralization of incentive efficient allocations as competitive equilibria in economies with asymmetric information. In a related paper (Jerez [18]) we build on the current analysis introducing firms, prices, and markets in the moral hazard economy in Section 3 and we present a notion of competitive equilibrium. Using the complementary slackness theorem, we then show that a competitive equilibrium exists and the welfare theorems hold. As the above discussion suggests, the case of adverse selection is more involved. However, we believe that the identification of the "net contribution to social welfare" of the different types in Section 2.5 might help to disentangle the external effects that arise with adverse selection and hence to understand how prices may or may not internalize such effects. If so, as in the case of moral hazard, the mechanics of linear programming may prove useful for the characterization of equilibrium prices and allocations.

\section{Acknowledgments}

This paper is part of my Ph.D. thesis and was revised while affiliated with the Universitat Autònoma de Barcelona. I am indebted to Joe Ostroy for his invaluable 
guidance and constant encouragement. I am very grateful to Alberto Bisin, Piero Gottardi, Andrés Erosa, David Levine, an associate editor and two referees for very helpful comments as well as Sushil Bikhchandani, Miquel Faig, Miguel A. Goberna, Birgit Grodal, Rong Li, Juan E. Martínez, Christine Neill, John Riley, Bill Zame, and participants of the ET Conference (Ischia), 8th World Congress of the Econometric Society (Seattle), SED Conference (Istanbul), European General Equilibrium Conference (Paris), European Winter Meeting of the Econometric Society (London), Istituto Veneto's Economic Theory Workshop (Venice), SITE at Stanford University, and seminars at UCLA, UBC, ITAM, Carlos III, UAB, Alicante, CERGE-EI and CORE. Financial support from the Alfred P. Sloan Dissertation Fellowship, the Bank of Spain, and the DGCYT under Projects PB980867 and FPI BEC2000-0172 is gratefully acknowledged. Errors are mine.

\section{Appendix A}

\section{A.1. The linear semi-infinite programming problems}

In this section, we set up the primal LSIP problem and derive its dual. Following Charnes et al. [7], we define the restricted dual problem, so-called dual problem in Haar's sense. The LSIP problems in Sections 2 and 3 obtain as particular cases of the problems in this section by applying the definitions in Table 1.

\section{A.1.1. The primal problem}

Let $1 \leqslant m \leqslant n$ and $\mathbb{R}^{n}$ be equipped with the Euclidean norm and partially ordered by means of the cone

$$
K_{m}^{n}=\left\{y \in \mathbb{R}^{n}: y_{j} \geqslant 0, j=1, \ldots, m\right\}
$$

Let $\omega \in \mathbb{R}_{+}^{2}$ and define $Z=\left\{z \in \mathbb{R}^{2}: z \geqslant-\omega\right\}$. Let $C(Z)$ denote the vector space of continuous real-valued functions on $Z$, endowed with the topology of uniform

Table 1

Adverse selection and moral hazard

\begin{tabular}{lll}
\hline & Adverse selection & Moral hazard \\
\hline$(n, m)$ & $(5,3)$ & $(4,3)$ \\
$y$ & $\left(\beta_{L}, \beta_{H}, q, \alpha_{L}, \alpha_{H}\right)$ & $\left(\beta_{L}, \beta_{H}, q, \alpha\right)$ \\
$c$ & $(0,0,0,1,1)$ & $(0,0,0,1)$ \\
$b=\left(b_{L}, b_{H}\right)$ & $\left(\gamma_{L} E U_{L},\left(1-\gamma_{L}\right) E U_{H}\right)$ & $\left(E U_{L}, E U_{H}\right)$ \\
$f_{1}=\left(f_{1 L}, f_{1 H}\right)$ & $\left(-E U_{L}, E U_{L}\right)$ & $\left(-E U_{L}+E U_{H}, 0\right)$ \\
$f_{2}=\left(f_{2 L}, f_{2 H}\right)$ & $\left(E U_{H},-E U_{H}\right)$ & $\left(0, E U_{L}-E U_{H}\right)$ \\
$f_{3}=\left(f_{3 L}, f_{3 H}\right)$ & $\left(\xi_{L} r_{L},\left(1-\xi_{L}\right) r_{H}\right)$ & $\left(r_{L}, r_{H}\right)$ \\
$f_{4}=\left(f_{4 L}, f_{4 H}\right)$ & $(\mathscr{I}, 0)$ & $(\mathscr{I}, \mathscr{I})$ \\
$f_{5}=\left(f_{5 L}, f_{5 H}\right)$ & $(0, \mathscr{I})$ & - \\
\hline
\end{tabular}


convergence on compact sets and partially ordered by means of the cone

$$
C_{+}(Z)=\{f \in C(Z): f(z) \geqslant 0 \quad \forall z \in Z\} .
$$

The primal problem is to find $y \in \mathbb{R}^{n}$ to solve

$$
\begin{array}{llc}
(P) \quad \text { inf } & c \cdot y \\
\text { s.t. } & A y \geqslant b, \\
& y \in K_{m}^{n},
\end{array}
$$

where $c \in \mathbb{R}^{n}, b=\left(b_{L}, b_{H}\right) \in C(Z) \times C(Z)$, and $A: \mathbb{R}^{n} \rightarrow C(Z) \times C(Z)$ is a continuous linear mapping. Problem $(P)$ is linear and has $n$ unknowns and infinitely many constraints. Denote its optimal value by $v(P)$.

\section{A.1.2. The dual problem}

Let $M_{\mathrm{c}}(Z)$ denote the space of signed Borel measures on $Z$ which have compact support and are finite on compact sets. This space is the topological dual space of $C(Z)$ (Hewitt [17]).

Let $C(Z) \times C(Z)$ be paired in duality with $M_{\mathrm{c}}(Z) \times M_{\mathrm{c}}(Z)$. The reflexive space $\mathbb{R}^{n}$ is paired with itself. The two pairings are endowed with their natural bilinear forms (to highlight the dimensionality of the spaces in the pairing we use the dot product and bracket notation for finite and infinite dimensions, respectively):

$$
\begin{gathered}
\langle f, x\rangle=\int_{Z} f_{L} d x_{L}+\int_{Z} f_{H} d x_{H}, \quad f=\left(f_{L}, f_{H}\right) \in C(Z) \times C(Z), \\
\quad x=\left(x_{L}, x_{H}\right) \in M_{\mathrm{c}}(Z) \times M_{\mathrm{c}}(Z), \\
y \cdot z=\sum_{j=1}^{n} y_{j} z_{j}, \quad y \in \mathbb{R}^{n}, \quad z \in \mathbb{R}^{n} .
\end{gathered}
$$

The adjoint of $A, A^{*}: M_{\mathrm{c}}(Z) \times M_{\mathrm{c}}(Z) \rightarrow \mathbb{R}^{n}$, is defined by the relation

$$
y \cdot\left(A^{*} x\right)=\langle A y, x\rangle, \text { for all } y \in K_{m}^{n}, x \in M_{\mathrm{c}_{+}}(Z) \times M_{\mathrm{c}_{+}}(Z) .
$$

We may write $A y=\sum_{j=1}^{n} y_{j} f_{j}$, where $f_{j}=\left(f_{j L}, f_{j H}\right) \in C(Z) \times C(Z)$ for $j=1, \ldots, n$. Then (A.1) can be expressed as

$$
y \cdot\left(A^{*} x\right)=\sum_{j=1}^{n} y_{j}\left\langle f_{j}, x\right\rangle, \quad \text { for all } y \in K_{m}^{n}, x \in M_{\mathrm{c}_{+}}(Z) \times M_{\mathrm{c}_{+}}(Z) .
$$

Write $A^{*} x \leqslant c$ as

$$
\sum_{j=1}^{n} y_{j}\left(\left\langle f_{j}, x\right\rangle-c_{j}\right) \leqslant 0, \quad \forall y \in K_{m}^{n}
$$


The dual problem is to find $x \in M_{\mathrm{c}}(Z) \times M_{\mathrm{c}}(Z)$ to solve

$$
\begin{aligned}
(D) \quad \sup & \langle b, x\rangle \\
\text { s.t. } & \left\langle f_{j}, x\right\rangle \leqslant c_{j}, \quad j=1, \ldots, m, \\
& \left\langle f_{j}, x\right\rangle=c_{j}, \quad j=m+1, \ldots, n, \\
& x \geqslant 0 .
\end{aligned}
$$

Problem $(D)$ is a linear programming problem with infinitely many unknowns and $n$ constraints. Denote its optimal value by $v(D)$. By the weak duality theorem (Krabs [21, Theorem I.3.1]), $v(D) \leqslant v(P)$.

\section{A.1.3. The dual problem in Haar's sense}

Let $\mathbb{R}^{(Z)}$ denote the vector space of all functions $\lambda_{i}: Z \rightarrow \mathbb{R}$ which vanish outside a finite subset of $Z$. For any $\lambda_{i} \in \mathbb{R}^{(Z)}$, we define the supporting set of $\lambda_{i}$ as

$$
\operatorname{supp} \lambda_{i}=\left\{z_{i} \in Z: \lambda_{i}\left(z_{i}\right) \neq 0\right\} \text {. }
$$

Let $C(Z) \times C(Z)$ be paired in duality with $\mathbb{R}^{(Z)} \times \mathbb{R}^{(Z)}$, with bilinear form

$$
\begin{aligned}
& \langle f, \lambda\rangle=\sum_{z_{L} \in \operatorname{supp} \lambda_{L}} f_{L}\left(z_{L}\right) \lambda_{L}\left(z_{L}\right)+\sum_{z_{H} \in \operatorname{supp} \lambda_{H}} f_{H}\left(z_{H}\right) \lambda_{H}\left(z_{H}\right), \\
& f=\left(f_{L}, f_{H}\right) \in C(Z) \times C(Z), \quad \lambda=\left(\lambda_{L}, \lambda_{H}\right) \in \mathbb{R}^{(Z)} \times \mathbb{R}^{(Z)} .
\end{aligned}
$$

The dual problem in Haar's sense is to find $\lambda \in \mathbb{R}^{(Z)} \times \mathbb{R}^{(Z)}$ to solve

$$
\begin{aligned}
\left(D_{\mathrm{F}}\right) \quad \sup & \langle b, \lambda\rangle \\
\text { s.t. } \quad & \left\langle f_{j}, \lambda\right\rangle \leqslant c_{j}, \quad j=1, \ldots, m, \\
& \left\langle f_{j}, \lambda\right\rangle=c_{j}, \quad j=m+1, \ldots, n, \\
& \lambda \geqslant 0 .
\end{aligned}
$$

Problem $\left(D_{\mathrm{F}}\right)$ is also a linear programming problem with infinitely many unknowns and $n$ constraints. Denote its optimal value by $v\left(D_{\mathrm{F}}\right)$. To see the relation between problems $(D)$ and $\left(D_{\mathrm{F}}\right)$ denote the set of finitely supported Borel measures on $Z$ by $M_{\mathrm{F}}$. Also, denote the Dirac measure at $z \in Z$ by $\delta_{z}$ (i.e., for any Borel set $B \subset Z$, $\delta_{z}(B)=1$ if $z \in B$ and $\delta_{z}(B)=0$ otherwise). Any pair $\lambda=\left(\lambda_{L}, \lambda_{H}\right) \in \mathbb{R}^{(Z)} \times \mathbb{R}^{(Z)}$ corresponds to a pair of finitely supported measures $x=\left(x_{L}, x_{H}\right)$ where $x_{i}=$ $\sum_{z_{i} \in \operatorname{supp} \lambda_{i}} \lambda_{i}\left(z_{i}\right) \delta_{z_{i}}$ for $i=L, H$. Thus, the space $\mathbb{R}^{(Z)} \times \mathbb{R}^{(Z)}$ is isomorphic to $M_{\mathrm{F}} \times$ $M_{\mathrm{F}}$. This implies that problem $\left(D_{\mathrm{F}}\right)$ is equivalent to problem $(D)$ when dual variables are restricted to lie in the subset $M_{\mathrm{F}} \times M_{\mathrm{F}}$ of $M_{\mathrm{c}}(Z) \times M_{\mathrm{c}}(Z)$, and so $v\left(D_{\mathrm{F}}\right) \leqslant v(D)$.

\section{A.2. Proofs of Theorems 2.2 and 2.1 and Proposition 2.1}

We begin with three preliminary Lemmas. To prove Lemma A.1 we appeal to the properties of the expected utility $E U_{i}$ and the expected net trade functions $r_{i}$ defined in Table 1. In particular, we use the continuity and strict concavity of $E U_{i}$, the fact 
that marginal utility of consumption decreases asymptotically to zero, and the linearity of $r_{i}$. The proof also uses the fact that at most one incentive constraint in problem $(D)$ binds with a positive shadow price. This property follows from Proposition 2.2 in the case of adverse selection, and is direct in the case of moral hazard since the incentive constraint (3.32) does not bind.

Lemma A.1. There exists a compact subset $T \subset Z$ such that, if all the constraints which are associated with elements $z \in Z \mid T$ are eliminated from problem $(P)$ then the set of optimal solutions does not change.

Proof. Let $Y$ denote the set of feasible solutions in problem $(P)$. That is, $y \in Y$ if and only if $y \in K_{m}^{n}$ and

$$
0 \geqslant h_{i}\left(z_{i}, y\right) \equiv b_{i}\left(z_{i}\right)-\sum_{j=1}^{n} y_{j} f_{j i}\left(z_{i}\right) \quad \forall z_{i} \in Z, \quad i=L, H
$$

(see Table 1). Note that $Y$ is a closed convex subset of $\mathbb{R}^{n}$. We establish the Lemma through a sequence of claims.

Claim 1: Y is non-empty.

Proof. This follows straightforwardly from (A.3) given the strict concavity of $b_{i}$ and the linearity of $f_{m i}$ for $i=L, H$ (see Table 1 ).

Claim 2: If $y \in Y$ then $y \geqslant 0$. Further, there exist constants $M_{j}, j=1, \ldots, n$, such that any optimal solution to problem $(P)$ lies in the set

$$
M=\left\{y \in Y: y_{j} \leqslant M_{j}, j=1, \ldots, n\right\} .
$$

Proof. By definition, any $y \in Y$ satisfies $y_{j} \geqslant 0$ for $j=1, \ldots, m$. Now, $f_{m i}(0)=0$ and utilities can be normalized so $b_{i}(0)=0$. Because $0 \in Z$, (A.3) implies that $y_{j} \geqslant 0$ for $j \geqslant m+1$. The existence of $M_{j}$ for $j \geqslant m+1$ follows from Claim 1 and the primal objective function. By the weak duality theorem and since autarky is a feasible solution for problem $(D)$,

$$
b_{L}(0)+b_{H}(0) \leqslant v(D) \leqslant v(P) \leqslant \sum_{j=m+1}^{n} M_{j} .
$$

Finally, since at most one incentive constraint has positive shadow price in problem $(D)$, it can be verified using (A.3) that at an optimal solution $y_{j}$ is bounded above for $1 \leqslant j \leqslant m$.

Claim 3: There is some $\varepsilon>0$ such that $y_{m}>\varepsilon$ for all $y \in M$.

Proof. Assume not. Then there exists a sequence $\left\{y^{k}\right\}$ in $M$ such that $0 \leqslant y_{m}^{k}<\frac{1}{k}$ for all $k \in \mathbb{N}$. Since at most one incentive constraint has positive shadow price, without 
loss of generality let $y_{1}=0$. Then, for some $i$ and any $y \in Y$,

$$
0 \geqslant h_{i}\left(z_{i}, y\right) \geqslant b_{i}\left(z_{i}\right)-y_{m} f_{m i}\left(z_{i}\right)-y_{n}, \quad \forall z_{i} \in Z .
$$

Since holds (A.4) for $y=y^{k}$, rearranging and taking limits gives

$$
\lim _{k \rightarrow \infty} y_{n}^{k} \geqslant b_{i}\left(z_{i}\right)-\lim _{k \rightarrow \infty} y_{m}^{k} f_{m i}\left(z_{i}\right)=b_{i}\left(z_{i}\right), \quad \forall z_{i} \in Z .
$$

Hence,

$$
\lim _{k \rightarrow \infty} y_{n}^{k} \geqslant b_{i}\left(z_{i}\right), \quad \forall z_{i} \in Z .
$$

When utility is unbounded, $\lim _{k \rightarrow \infty} y_{m+1}^{k}=\infty$, thereby contradicting Claim 2 . When utility is bounded, $\lim _{z_{i} \rightarrow \infty} b_{i}\left(z_{i}\right)=B_{i}$. But $M_{n}$ can then always be found in $\left(0, B_{i}\right)$, leading to a similar contradiction.

Claim 4: There is a $\bar{z}$ such that, for each $i=L, H$ and each $y \in M, \nabla h_{i}\left(z_{i}, y\right) \ll 0$ for all $z_{i}>\bar{z}$.

Proof. Without loss of generality, take $i=L$. From Table $1, \nabla f_{j L}=0$ for $j \geqslant m+1$. Also, $\nabla f_{m L}\left(z_{L}\right)=\bar{g}_{L} \gg 0$. Then

$$
\begin{aligned}
\nabla h_{L}\left(z_{L}, y\right) & =\nabla b_{L}\left(z_{L}\right)-\sum_{j=1}^{m} y_{j} \nabla f_{j L}\left(z_{L}\right) \\
& =\nabla b_{L}\left(z_{L}\right)-\sum_{j=1}^{m-1} y_{j}\left(\nabla f_{j L}^{+}\left(z_{L}\right)-\nabla f_{j L}^{-}\left(z_{L}\right)\right)-y_{m} \bar{g}_{L},
\end{aligned}
$$

where $\nabla f_{j L}^{+}$and $\nabla f_{j L}^{-} \geqslant 0$ stand for the positive and negative parts of $\nabla f_{j L}$. This together with Claims 2 and 3 implies

$$
\nabla h_{L}\left(z_{L}, y\right) \leqslant \nabla b_{L}\left(z_{L}\right)+\sum_{j=1}^{m-1} M_{j} \nabla f_{j L}^{-}\left(z_{L}\right)-\varepsilon \bar{g}_{L}, \quad \forall z_{L} \in Z .
$$

But as marginal utility decreases asymptotically to zero:

$$
\begin{aligned}
& \lim _{z_{L} \rightarrow+\infty} \nabla b_{L}\left(z_{L}\right)=0, \\
& \lim _{z_{L} \rightarrow+\infty} \nabla f_{j L}\left(z_{L}\right)=0, \quad 1 \leqslant j \leqslant m-1,
\end{aligned}
$$

and this gives

$$
\lim _{z_{L} \rightarrow+\infty} \nabla h_{L}\left(z_{L}, y\right):::-\varepsilon \bar{g}_{L} \ll 0 .
$$

Since $h_{L}(\cdot, y)$ is a continuously differentiable, there exists a constant $\bar{z}_{L}$ such that $\nabla h_{L}\left(z_{L}, y\right) \ll 0$ for all $z_{L}>\bar{z}_{L}$. A similar derivation gives $\bar{z}_{H}$. Setting $\bar{z}=\max \left\{\bar{z}_{L}, \bar{z}_{H}\right\}$ proves our claim.

Claim 5: The set $T=\left[-\omega_{1}, \bar{z}\right] \times\left[-\omega_{2}, \bar{z}\right]$ satisfies Lemma A.1. 
Proof. Claim 5 is direct from Claim 4. This completes the proof of Lemma A.1.

Consider the LSIP problems which arise by replacing $Z$ by $T$ in problems $(P),(D)$ and $\left(D_{\mathrm{F}}\right)$. Denote these problems by $\left(P^{T}\right),\left(D^{T}\right)$ and $\left(D_{\mathrm{F}}^{T}\right)$, respectively. The proofs of Lemmas A.2 and A.3 below appeal to some well-known results in LSIP theory. The proof of Lemma A.2 exploits also the strict concavity of $E U_{i}$ and the linearity of $r_{i}$.

Lemma A.2. The system of constraints in problem $\left(P^{T}\right)$ is canonically closed in the sense of Charnes et al. [8].

Proof. First, since $T$ is compact and since $b_{i}$ and $f_{j i}$ are continuous for all $i$ and $j$, the set

$$
\left\{\left(f_{1}(t), f_{2}(t), \ldots, f_{n}(t), b(t)\right): t \in T\right\}
$$

is compact in $\mathbb{R}^{n+1}$.

Second, the Slater constraint qualification is satisfied. To see this, let $y_{j}^{0}=0$ for $1 \leqslant j \leqslant m-1$ and let $y_{m}^{0}>0$ be given. Since, $f_{m i}$ is linear and $b_{i}$ is strictly concave, there exist constants $a_{L}>0$ and $a_{H}>0$ and values for $y_{j}^{0}$ for $j \geqslant m+1$ such that,

$$
\begin{array}{ll}
0 \geqslant h_{L}\left(z_{L}, y^{0}\right)=b_{L}\left(z_{L}\right)-y_{m}^{0} f_{m L}\left(z_{L}\right)-y_{m+1}^{0}+a_{L}, & \forall z_{L} \in Z, \\
0 \geqslant h_{H}\left(z_{H}, y^{0}\right)=b_{H}\left(z_{H}\right)-y_{m}^{0} f_{m H}\left(z_{H}\right)-y_{n}^{0}+a_{H}, \quad \forall z_{H} \in Z .
\end{array}
$$

That is, $y^{0}$ is a Slater point.

Lemma A.3. Problem $\left(D_{\mathrm{F}}^{T}\right)$ is solvable and $v\left(D_{\mathrm{F}}^{T}\right)=v\left(D^{T}\right)=v\left(P^{T}\right)$.

Proof. By weak duality of the pair $\left\{\left(P^{T}, D^{T}\right)\right\}$, and the definition of $\left(D_{\mathrm{F}}^{T}\right)$,

$$
v\left(D_{\mathrm{F}}^{T}\right) \leqslant v\left(D^{T}\right) \leqslant v\left(P^{T}\right) .
$$

Given Lemma A.2, the inhomogeneous Haar theorem of Charnes et al. [7, Theorem 3] implies that the system of constraints in $\left(P^{T}\right)$ has the Farkas-Minkoswki property. Since $\left(P^{T}\right)$ and $\left(D_{\mathrm{F}}^{T}\right)$ are consistent, the extended dual theorem of Charnes et al. [7, Theorem 4] implies then that $\left(D_{\mathrm{F}}^{T}\right)$ is solvable and that $v\left(D_{\mathrm{F}}^{T}\right)=v\left(P^{T}\right)$.

We are now ready to prove Theorems 2.1 and 2.2, and Proposition 2.1.

Proof of Theorem 2.1. By weak duality of the pair $\{(P),(D)\}$, and the definition of $\left(D_{\mathrm{F}}\right)$,

$$
v\left(D_{\mathrm{F}}\right) \leqslant v(D) \leqslant v(P) .
$$

Also, since $\mathbb{R}^{(T)} \subset \mathbb{R}^{(Z)}$, it follows that $v\left(D_{\mathrm{F}}^{T}\right) \leqslant v\left(D_{\mathrm{F}}\right)$. By Lemma A.1, $v(P)=v\left(P^{T}\right)$. But then, Lemma A.3 implies that $v\left(D_{\mathrm{F}}\right)=v(D)=v(P)$. 
Proof of Proposition 2.1. The proof of Theorem 2.1 establishes that $v\left(D_{\mathrm{F}}\right)=v(D)$. It also implies that $v\left(D_{\mathrm{F}}^{T}\right)=v\left(D_{\mathrm{F}}\right)$. Since by Lemma A.3, $\left(D_{\mathrm{F}}^{T}\right)$ is solvable, so is $\left(D_{\mathrm{F}}\right)$.

Proof of Theorem 2.2. The solvability of $(D)$ follows from Proposition 2.1. By Claims 1 and 2 in Lemma A.1, $Y$ is non-empty and may be assumed bounded. Since $Y$ is closed, problem $(P)$ maximizes a continuous function on a compact set, and so its value is attained.

\section{A.3. Proofs of Propositions 2.2, 2.4-2.6, and 3.4-3.5}

Proof of Proposition 2.2. Let $\left(x^{*}, x^{*}\right)$ denote the first best equal treatment allocation: $x^{*}=\delta_{z^{*}}$ with $z^{*}=\left(z_{1}^{*}, z_{2}^{*}\right)$ and $z_{s}^{*}=\bar{\omega}-\omega_{s}$ for $s=1,2$. Since $\left(x^{*}, x^{*}\right)$ is incentive compatible, it is also incentive efficient. Let $\bar{\gamma}_{L}$ and $\bar{\gamma}_{H}$ be the associated weights in problem $(D)$. Using first-order conditions,

$$
v_{i}^{\prime}\left(z^{*} ; q^{*}\right)=\bar{\gamma}_{i} U_{i}^{\prime}(\bar{\omega})-\xi_{i} q^{*}=0, \quad i=L, H .
$$

Writing $\bar{\gamma}_{H}=1-\bar{\gamma}_{L}$ and rearranging gives

$$
\bar{\gamma}_{L}=\left(1+\frac{\left(1-\xi_{L}\right) U_{L}^{\prime}(\bar{\omega})}{\xi_{L} U_{H}^{\prime}(\bar{\omega})}\right)^{-1} .
$$

Any other incentive efficient allocation $\left(x_{L}^{*}, x_{H}^{*}\right)$ is such that either (i) one type is strictly better off, or (ii) both types are indifferent. Assume (i) and suppose, without loss of generality, that type $L$ is better off. Then, $\gamma_{L}>\bar{\gamma}_{L}$. Since $x^{*}$ provides full insurance, the expected consumption of type $L$ must exceed $\bar{\omega}$ and, by feasibility, that of type $H$ must be lower than $\bar{\omega}$. But then,

$$
\left\langle U_{L}, x_{H}^{*}\right\rangle\left\langle\langle U _ { L } , x ^ { * } \rangle \left\langle\left\langle U_{L}, x_{L}^{*}\right\rangle,\right.\right.
$$

so the incentive constraint of type $L$ is does not bind $\left(\beta_{L}=0\right)$. Since the incentive constraint of type $H$ is satisfied, $x_{L}^{*}$ entails only partial insurance. This constraint must bind with $\beta_{H}>0$; otherwise, the utility of type $L$ could be increased by reducing the risk in $x_{L}^{*}$ and maintaining the expected consumption.

Case (ii) is impossible. If each type $i$ is indifferent between $x_{i}^{*}$ and $x^{*}$ then $\left(x_{L}^{*}, x_{H}^{*}\right)$ must give both types an expected consumption of at least $\bar{\omega}$. But, by feasibility, the expected consumption of both types must equal $\bar{\omega}$. Since $\left(x_{L}^{*}, x_{H}^{*}\right)$ and $\left(x^{*}, x^{*}\right)$ are different, at least one type $i$ is not fully insured and strictly prefers $x^{*}$ to $x_{i}^{*}$, a contradiction.

Proof of Proposition 2.4. We first show that, when $U_{i}(\cdot)=U(\cdot)$ for $i=L, H$, the function $v_{L}\left(\cdot ; \beta_{H}^{*}, q^{*}\right)$ is strictly concave. This function is additive across states,

$$
v_{L}\left(z_{L} ; \beta_{H}^{*}, q^{*}\right)=\sum_{s \in\{1,2\}} v_{L s}\left(z_{L s} ; \beta_{H}^{*}, q^{*}\right),
$$


where

$$
\begin{aligned}
& v_{L 1}\left(z_{L 1} ; \beta_{H}^{*}, q^{*}\right)=\left(\gamma_{L} \theta_{L}-\beta_{H}^{*} \theta_{H}\right) U\left(w_{1}+z_{L 1}\right)-q^{*} \xi_{L} \theta_{L} z_{L 1}, \\
& v_{L 2}\left(z_{L 2} ; \beta_{H}^{*}, q^{*}\right)=\left(\gamma_{L}\left(1-\theta_{L}\right)-\beta_{H}^{*}\left(1-\theta_{H}\right)\right) U\left(w_{2}+z_{L 2}\right)-q^{*} \xi_{L}\left(1-\theta_{L}\right) z_{L 2} .
\end{aligned}
$$

Since $U^{\prime \prime}<0$, for $s=1,2$, the second derivative $v_{L s}^{\prime \prime}$ never changes sign. Suppose $v_{L s}^{\prime \prime} \geqslant 0$ for some $s$. Because $U^{\prime}>0$, then $v_{L s}^{\prime}<0$. But then, by condition (2.27), the optimal assignment to type $L$ is deterministic and such that $w_{s}+z_{L s}^{*}=0$, which is impossible since $\lim _{c \rightarrow 0} U_{L}^{\prime}(0)=\infty$. We conclude that $v_{L s}^{\prime \prime}<0$ for $s=1,2$.

Since $0<\theta_{L}<\theta_{H}<1$ and $U^{\prime \prime}<0$, the first-order conditions imply that the maximum of $v_{L}\left(\cdot ; \beta_{H}^{*}, q^{*}\right)$ satisfies $\omega_{1}+z_{L 1}<\omega_{2}+z_{L 2}$.

Proof of Proposition 2.5. We may write

$$
v_{L}\left(z_{L} ; \beta_{H}^{*}, q^{*}\right)=\sum_{s \in\{1,2\}} v_{L s}\left(z_{L s} ; \beta_{H}^{*}, q^{*}\right),
$$

where

$$
\begin{aligned}
v_{L 1}\left(z_{L 1} ; \beta_{H}^{*}, q^{*}\right)= & \gamma_{L} \theta_{L} U_{L}\left(w_{1}+z_{L 1}\right)-\beta_{H}^{*} \theta_{H} U_{H}\left(w_{1}+z_{L 1}\right)-q^{*} \xi_{L} \theta_{L} z_{L 1}, \\
v_{L 2}\left(z_{L 2} ; \beta_{H}^{*}, q^{*}\right)= & \gamma_{L}\left(1-\theta_{L}\right) U_{L}\left(w_{2}+z_{L 2}\right)-\beta_{H}^{*}\left(1-\theta_{H}\right) U_{H}\left(w_{2}+z_{L 2}\right) \\
& -q^{*} \xi_{L}\left(1-\theta_{L}\right) z_{L 2} .
\end{aligned}
$$

We first show that, if $A_{L}(c) \geqslant A_{H}(c)$ for all $c \in \mathbb{R}_{+}$then $v_{L}\left(\cdot ; \beta_{H}^{*}, q^{*}\right)$ is strictly concave. Write $v_{L 1}^{\prime}=\left(g_{1}+g_{2}\right) g_{3}$ where

$$
g_{1}=\gamma_{L} \theta_{L} \frac{U_{L}^{\prime}}{U_{H}^{\prime}}, \quad g_{2}=-\left(\beta_{H}^{*} \theta_{H}+\frac{q^{*} \xi_{L} \theta_{L}}{U_{H}^{\prime}}\right), \quad g_{3}=U_{H}^{\prime} .
$$

Clearly, $g_{2}^{\prime}, g_{3}^{\prime}<0$. Further,

$$
\frac{1}{\gamma_{L} \theta_{L}} g_{1}^{\prime}=\left(\frac{U_{L}^{\prime \prime} U_{H}^{\prime}-U_{L}^{\prime} U_{H}^{\prime \prime}}{\left(U_{H}^{\prime}\right)^{2}}\right)=\left(\frac{\left(\frac{U_{L}^{\prime \prime} U_{H}^{\prime}}{U_{L}^{\prime} U_{H}^{\prime \prime}}-1\right) U_{L}^{\prime} U_{H}^{\prime \prime}}{\left(U_{H}^{\prime}\right)^{2}}\right)=\left(\frac{\left(\frac{A_{L}}{A_{H}}-1\right) U_{L}^{\prime} U_{H}^{\prime \prime}}{\left(U_{H}^{\prime}\right)^{2}}\right) .
$$

So $A_{L} \geqslant A_{H}$ implies $g_{1}^{\prime} \leqslant 0$ and hence $v_{L 1}^{\prime \prime}<0$. Finally, because $0<\theta_{L}<\theta_{H}<1, v_{L 1}^{\prime \prime}<0$ implies $v_{L 2}^{\prime \prime}<0$, which proves our claim.

Now $g_{1}^{\prime} \leqslant 0$ is equivalent to $\frac{U_{L}^{\prime}}{U_{H}^{\prime}}$ being non-increasing. Since $0<\theta_{L}<\theta_{H}<1$ and $U^{\prime \prime}<0$, the first-order conditions imply that the maximum of $v_{L}\left(\cdot ; \beta_{H}^{*}, q^{*}\right)$ satisfies $\omega_{1}+z_{L 1}<\omega_{2}+z_{L 2}$.

Proof of Proposition 2.6. For $\gamma_{L} \in\left(0, \bar{\gamma}_{L}\right)$, the net contributions are:

$$
\begin{aligned}
& v_{L}\left(z_{L} ; \beta_{L}, q\right)=\gamma_{L} E U_{L}\left(z_{L}\right)-q \xi_{L} r_{L}\left(z_{L}\right)+\beta_{L} E U_{L}\left(z_{L}\right), \\
& v_{H}\left(z_{H} ; \beta_{L}, q\right)=\left(1-\gamma_{L}\right) E U_{H}\left(z_{H}\right)-q\left(1-\xi_{L}\right) r_{H}\left(z_{H}\right)-\beta_{L} E U_{L}\left(z_{L}\right) .
\end{aligned}
$$

Similar arguments to those in the proofs of Propositions 2.3, 2.4, and 2.5 prove (i) and (ii). 
Proof of Proposition 3.2. Define $U(\cdot)=U_{H}(\cdot)$, so $U_{L}(\cdot)=U(\cdot)+d$. Then

$$
v_{H}\left(z_{H} ; \beta_{H}^{*}, q^{*}\right)=\sum_{s \in\{1,2\}} v_{H s}\left(z_{H s} ; \beta_{H}^{*}, q^{*}\right),
$$

where

$$
\begin{aligned}
v_{H 1}\left(z_{H 1} ; \beta_{H}^{*}, q^{*}\right)= & \left(\left(1+\beta_{H}^{*}\right) \theta_{H}-\beta_{H}^{*} \theta_{L}\right) U\left(w_{1}+z_{H 1}\right)-q^{*} \theta_{H} z_{H 1}-\beta_{H}^{*} \theta_{L} d, \\
v_{H 2}\left(z_{H 2} ; \beta_{H}^{*}, q^{*}\right)= & \left(\left(1+\beta_{H}^{*}\right)\left(1-\theta_{H}\right)-\beta_{H}^{*}\left(1-\theta_{L}\right)\right) U\left(w_{2}+z_{H 2}\right) \\
& -q^{*}\left(1-\theta_{H}\right) z_{H 2}-\beta_{H}^{*}\left(1-\theta_{L}\right) d .
\end{aligned}
$$

Analogous arguments to those in the proof of Proposition 2.4 show that $v_{H}\left(\cdot ; \beta_{H}^{*}, q^{*}\right)$ is strictly concave and that its maximum is characterized by partial insurance.

The proof of Proposition 3.3 is analogous to that of Proposition 2.5 and is omitted.

Proof of Proposition 3.4. Take an arbitrary endowment sequence $\left\{\omega^{k}\right\} \subset \mathbb{R}_{+}^{2}$ such that $\lim _{k \rightarrow \infty} \bar{\omega}_{H}^{k}=\infty$ and $\bar{\omega}_{H}^{k}-\bar{\omega}_{L}^{k} \leqslant N$ for some constant $N$ (with $\bar{\omega}_{i}^{k}$ denoting the average endowment with effort $e_{i}$ when $\left.\omega=\omega^{k}\right)$. Let $\left(\alpha^{k}, \beta_{H}^{k}, q^{k}\right)$ and $\left(x_{L}^{k}, x_{H}^{k}\right)$ be optimal primal and dual solutions for $\omega=\omega^{k}$.

Suppose $\left\|x_{H}^{k}\right\|=1$ for all $k$. Since $q^{k}>0$, by (3.47), the support of $x_{H}^{k}$ becomes unbounded as $k$ increases. In particular, since $\beta_{H}^{k}>0$, by (3.46), there is a sequence $\left\{z_{H}^{k}\right\}$ where $z_{H}^{k}=\left(z_{H 1}^{k}, z_{H 2}^{k}\right)$ such that $x_{H}^{k}\left(z_{H}^{k}\right)>0$ and $\lim _{k \rightarrow \infty} z_{H 2}^{k}=\infty$. Write the first-order condition associated to (3.48) for $s=2$ as

$$
\left(\frac{1-\theta_{L}}{1-\theta_{H}}-\frac{U_{H}^{\prime}\left(w_{2}^{k}+z_{H 2}^{k}\right)}{U_{L}^{\prime}\left(w_{2}^{k}+z_{H 2}^{k}\right)}\right) \beta_{H}^{k}=\frac{U_{H}^{\prime}\left(w_{2}^{k}+z_{H 2}^{k}\right)}{U_{L}^{\prime}\left(w_{2}^{k}+z_{H 2}^{k}\right)}-\frac{q^{k}}{U_{L}^{\prime}\left(w_{2}^{k}+z_{H 2}^{k}\right)} .
$$

It can be checked from conditions (3.46) and (3.48), and definition (3.42) that the sequence $\left\{\beta_{H}^{k}\right\}$ must be bounded. Since $U_{H}^{\prime} \leqslant U_{L}^{\prime}$, the left-hand side of (A.5) is bounded below, and the first term on the right-hand side of (A.5) is bounded above. Thus, since $\lim _{c \rightarrow \infty} U_{L}^{\prime}(c)=0$, (A.5) implies that $\lim _{k \rightarrow \infty} q^{k}=0$.

Let $v_{L}^{k}\left(q^{k}\right)$ and $v_{H}^{k}\left(\beta_{H}^{k}, q^{k}\right)$ denote the maximal net contributions with low and high effort for $\omega=\omega^{k}$. Using (3.48) and the equality of the optimal primal and dual values,

$$
\alpha^{k}=v_{H}^{k}\left(\beta_{H}^{k}, q^{k}\right)=\left\langle E U_{H}, x_{H}^{k}\right\rangle .
$$

Define the certainty equivalent $\bar{c}_{H}^{k}$ associated to $x_{H}^{k}$, so $U_{H}\left(\bar{c}_{H}^{k}\right)=\left\langle E U_{H}, x_{H}^{k}\right\rangle$. Since $U_{H}^{\prime \prime}<0$, then $\bar{c}_{H}^{k}<\bar{\omega}_{H}^{k}$. Applying (3.43) for $z_{L s}^{k}=\bar{c}_{H}^{k}-w_{s}^{k}, s=1,2$, gives

$$
v_{L}^{k}\left(q^{k}\right) \geqslant U_{L}\left(\bar{c}_{H}^{k}\right)-q^{k}\left(\bar{c}_{H}^{k}-\bar{\omega}_{L}^{k}\right)>U_{L}\left(\bar{c}_{H}^{k}\right)-q^{k}\left(\bar{\omega}_{H}^{k}-\bar{\omega}_{L}^{k}\right) .
$$

Since $\lim _{k \rightarrow \infty} q^{k}=0$ and $\lim _{k \rightarrow \infty}\left(\bar{\omega}_{H}^{k}-\bar{\omega}_{L}^{k}\right) \leqslant N$, (A.7) implies that for any $\varepsilon>0$ there is $K$ such that $U_{L}\left(\bar{c}_{H}^{k}\right)-v_{L}^{k}\left(q^{k}\right) \leqslant \varepsilon$ for all $k \geqslant K$. Fix $\varepsilon \leqslant d$. Because $U_{H}^{\prime \prime}<0$ and 
$U_{L}(\cdot)-U_{H}(\cdot)=d,($ A.6) implies

$$
\alpha^{k}=\left\langle E U_{H}, x_{H}^{k}\right\rangle<U_{H}\left(\bar{c}_{H}^{k}\right)=U_{L}\left(\bar{c}_{H}^{k}\right)-d
$$

and, since $\left\|x_{L}^{k}\right\|=0,(3.48)$ implies

$$
\alpha^{k}>v_{L}^{k}\left(q^{k}\right)
$$

Eqs. (A.8) and (A.9) imply that $U_{L}\left(\bar{c}_{H}^{k}\right)-v_{L}^{k}\left(q^{k}\right)>d$, a contradiction for $k \geqslant K$. We conclude that $\left\|x_{H}^{k}\right\|<1$ for all $k \geqslant K$.

Proof of Proposition 3.5. Suppose we restrict the allocations to satisfy $x_{L}=0$. Take any endowment sequence $\left\{\omega^{k}\right\} \subset \mathbb{R}_{+}^{2}$ with $\lim _{k \rightarrow \infty} \bar{\omega}_{H}^{k}=\infty$. Let $\left(\hat{\beta}_{H}^{k}, \hat{q}^{k}\right)$ and $\hat{x}_{H}^{k}$ be optimal primal and dual solutions to the restricted problem for $\omega=\omega_{k}$. The complementary slackness conditions are obtained by letting $x_{L}=0$ in (3.46)-(3.48).

Suppose, in contrast to what we want to show, that $\left\langle r_{H}, \hat{x}_{H}^{k}\right\rangle=0$ for all $k$. Since (3.46) holds, there is a sequence $\left\{z_{H}^{k}\right\}$ where $z_{H}^{k}=\left(z_{H 1}^{k}, z_{H 2}^{k}\right)$ such that $\hat{x}_{H}^{k}\left(z_{H}^{k}\right)>0$, $\lim _{k \rightarrow \infty} z_{H 2}^{k}=\infty$, and $\left(\omega_{H 2}+z_{H 2}^{k}-\omega_{H 1}-z_{H 1}^{k}\right) \geqslant \varepsilon_{1}$ for some $\varepsilon_{1}>0$ and all $k$. Write the first-order conditions associated to (3.48) as:

$$
\begin{aligned}
& \left(\frac{\theta_{L}}{\theta_{H}}-\frac{U_{H}^{\prime}\left(w_{1}^{k}+z_{H 1}^{k}\right)}{U_{L}^{\prime}\left(w_{1}^{k}+z_{H 1}^{k}\right)}\right) \hat{\beta}_{H}^{k}=\frac{U_{H}^{\prime}\left(w_{1}^{k}+z_{H 1}^{k}\right)}{U_{L}^{\prime}\left(w_{1}^{k}+z_{H 1}^{k}\right)}-\frac{\hat{q}^{k}}{U_{L}^{\prime}\left(w_{1}^{k}+z_{H 1}^{k}\right)}, \\
& \left(\frac{1-\theta_{L}}{1-\theta_{H}}-\frac{U_{H}^{\prime}\left(w_{2}^{k}+z_{H 2}^{k}\right)}{U_{L}^{\prime}\left(w_{2}^{k}+z_{H 2}^{k}\right)}\right) \hat{\beta}_{H}^{k}=\frac{U_{H}^{\prime}\left(w_{2}^{k}+z_{H 2}^{k}\right)}{U_{L}^{\prime}\left(w_{2}^{k}+z_{H 2}^{k}\right)}-\frac{\hat{q}^{k}}{U_{L}^{\prime}\left(w_{2}^{k}+z_{H 2}^{k}\right)} .
\end{aligned}
$$

Since $\lim _{c \rightarrow \infty} \frac{U_{H}^{\prime}(c)}{U_{L}^{\prime}(c)}=0$, taking limits in (A.11) yields

$$
\left(\frac{1-\theta_{L}}{1-\theta_{H}}\right) \lim _{k \rightarrow \infty} \hat{\beta}_{H}^{k}=-\lim _{k \rightarrow \infty} \frac{\hat{q}^{k}}{U_{L}^{\prime}\left(w_{2}^{k}+z_{H 2}^{k}\right)},
$$

so $\lim _{k \rightarrow \infty} \hat{\beta}_{H}^{k}=\lim _{k \rightarrow \infty} \hat{q}^{k}=0$. Now, for $k$ sufficiently large, the right-hand side of (A.11) is positive, so $\hat{q}^{k}<U_{H}^{\prime}\left(w_{2}^{k}+z_{H 2}^{k}\right)$. Since $U_{L}^{\prime \prime}<0$ and $\omega_{H 2}+z_{H 2}^{k}>\omega_{H 1}-z_{H 1}^{k}$, (A.10) implies

$$
\left(\frac{\theta_{L}}{\theta_{H}}-\frac{U_{H}^{\prime}\left(w_{1}^{k}+z_{H 1}^{k}\right)}{U_{L}^{\prime}\left(w_{1}^{k}+z_{H 1}^{k}\right)}\right) \hat{\beta}_{H}^{k}>\frac{U_{H}^{\prime}\left(w_{1}^{k}+z_{H 1}^{k}\right)}{U_{L}^{\prime}\left(w_{1}^{k}+z_{H 1}^{k}\right)}-\frac{U_{H}^{\prime}\left(w_{2}^{k}+z_{H 2}^{k}\right)}{U_{L}^{\prime}\left(w_{2}^{k}+z_{H 2}^{k}\right)} .
$$


But as $U_{i}$ is continuously differentiable and $\left(\omega_{H 2}+z_{H 2}^{k}-\omega_{H 1}-z_{H 1}^{k}\right) \geqslant \varepsilon_{1}$, taking limits and rearranging in (A.12) gives

$$
\lim _{k \rightarrow \infty} \hat{\beta}_{H}^{k}>\left(\frac{\theta_{L}}{\theta_{H}}\right)^{-1} \lim _{k \rightarrow \infty}\left(\frac{U_{H}^{\prime}\left(w_{1}^{k}+z_{H 1}^{k}\right)}{U_{L}^{\prime}\left(w_{1}^{k}+z_{H 1}^{k}\right)}-\frac{U_{H}^{\prime}\left(w_{2}^{k}+z_{H 2}^{k}\right)}{U_{L}^{\prime}\left(w_{2}^{k}+z_{H 2}^{k}\right)}\right) \geqslant\left(\frac{\theta_{L}}{\theta_{H}}\right)^{-1} \varepsilon_{2},
$$

for some constant $\varepsilon_{2}>0$, a contradiction.

\section{References}

[1] R. Arnott, B.C. Greenwald, J.E. Stiglitz, Information and economic efficiency, Inform. Econom. Polit. 6 (1994) 77-88.

[2] R. Arnott, J.E. Stiglitz, Randomization with asymmetric information, RAND J. Econom. 19 (1988) 344-362.

[3] A. Bennardo, P.A. Chiappori, Bertrand and Walras equilibria under moral hazard, Mimeo, 2000.

[4] A. Bisin, P. Gottardi, Competitive equilibria with asymmetric information, J. Econom. Theory 87 (1999) 1-48.

[5] A. Bisin, P. Gottardi, Decentralizing incentive efficient allocations of economies with adverse selection, Mimeo, 2000.

[6] A. Bisin, D. Guatoli, Inefficiency of competitive equilibrium with asymmetric information and financial intermediaries, CEPR Discussion Paper 1987, 1997.

[7] A. Charnes, W.W. Cooper, K. Kortanek, Duality in semi-infinite programs and some works of Haar and Carathédory, Management Sci. 9 (1963) 209-228.

[8] A. Charnes, W.W. Cooper, K. Kortanek, On representations of semi-infinite programs which have no duality gaps, Management Sci. 2 (1965) 113-121.

[9] H.L. Cole, Comment: general competitive analysis in an economy with asymmetric information, Int. Econom. Rev. 30 (1989) 249-253.

[10] P. Dubey, J. Geanakoplos, M. Shubik, Default and efficiency in a general equilibrium model with incomplete markets, Mimeo, 1995.

[11] M. Feldman, C. Gilles, An expository note on individual risk without aggregate uncertainty, J. Econom. Theory 35 (1985) 26-32.

[12] M.A. Goberna, M.A. López, Linear Semi-Infinite Optimization, Wiley, Chichester, 1998.

[13] B.C. Greenwald, J.E. Stiglitz, Externalities in economies with imperfect information and incomplete markets, Quart. J. Econom. 101 (1986) 229-264.

[14] N.E. Gretsky, J.M. Ostroy, W.R. Zame, Perfect competition in the continuous assignment model, J. Econom. Theory 88 (1999) 60-118.

[15] P.J. Hammond, M.B. Lisboa, Monte Carlo integration and an exact law of large numbers for a continuum of independent random variables, Mimeo, 1998.

[16] M. Harris, R.M. Townsend, Resource allocation under asymmetric information, Econometrica 49 (1981) 33-64 Mimeo, 1998.

[17] E. Hewitt, Linear functionals on spaces of continuous functions, Fundam. Math. 37 (1959) 161-189.

[18] B. Jerez, General equilibrium with asymmetric information: a dual approach, UAB Working Paper $510.02,2000$.

[19] K. Judge, The law of large numbers with a continuum of iid random variables, J. Econom. Theory 35 (1985) 19-25.

[20] T.J. Kehoe, D.K. Levine, E. Prescott, Lotteries, sunspots and incentive constraints, J. Econom. Theory, doi:10.1006/jeth.2001.2792.

[21] W. Krabs, Optimization and Approximation, Wiley, New York, 1979. 
[22] L. Makowski, J.M. Ostroy, Perfect competition via linear programming, Mimeo, 1996.

[23] R.B. Myerson, Incentive compatibility and the bargaining problem, Econometrica 47 (1979) 61-74.

[24] R.B. Myerson, Optimal coordinating mechanisms in generalized principal-agent problems, J. Math. Econom. 10 (1982) 67-81.

[25] R.B. Myerson, Cooperative games with incomplete information, Internat. J. Game Theory 13 (1984) 69-96.

[26] E. Prescott, R. Townsend, Pareto optima and competitive equilibria with adverse selection and moral hazard, Econometrica 52 (1984) 21-45.

[27] E. Prescott, R. Townsend, General competitive analysis in an economy with private information, Int. Econom. Rev. 25 (1984) 1-20.

[28] M. Rothschild, J.E. Stiglitz, Equilibrium in competitive insurance markets: an essay on the economics of imperfect information, Quart. J. Econom. 90 (1976) 629-649.

[29] Y. Sun, A theory of hyperfinite processes: the complete removal of individual uncertainty via exact LLN, J. Math. Econom. 29 (1998) 419-503.

[30] C. Wilson, A model of insurance markets with incomplete information, J. Econom. Theory 16 (1977) 176-207. 